FZJ-IKP(TH)-2002-12

\title{
Near threshold neutral pion electroproduction on deuterium in chiral perturbation theory
}

\author{
H. Krebs ${ }^{1}$, V. Bernard ${ }^{2}$, Ulf-G. Meißner ${ }^{1,3}$ \\ ${ }^{1}$ Institut für Kernphysik (Theorie), Forschungszentrum Jülich \\ D-52425 Jülich, Germany \\ E-mail addresses: h.krebs@fz-juelich.de,u.meissner@fz-juelich.de \\ ${ }^{2}$ Laboratoire de Physique Théorique, Université Louis Pasteur \\ F-67037 Strasbourg Cedex 2, France \\ E-mail address: bernard@lpt6.u-strasbg.fr \\ ${ }^{3}$ Institut für Theoretische Physik, Karl-Franzens-Universität Graz \\ A-8010 Graz, Austria
}

\begin{abstract}
Near threshold neutral pion electroproduction on the deuteron is studied in the framework of baryon chiral perturbation theory at next-to-leading order in the chiral expansion. We develop the multipole decomposition for pion production off spin-1 particles appropriate for the threshold region. The existing data at photon virtuality $k^{2}=-0.1 \mathrm{GeV}^{2}$ can be described satisfactorily. Furthermore, the prediction for the S-wave multipole $E_{d}$ at the photon point is in good agreement with the data.
\end{abstract}

PACS nos.: 25.20.Lj , 12.39.Fe

Keywords: Pion electroproduction, deuteron, chiral perturbation theory

${ }^{\# 1}$ Work supported in part by Deutsche Forschungsgemeinschaft under contract no. Me864-16/2. 


\section{Introduction}

Pion photo- and electroproduction off single nucleons in the threshold region can be considered one of the best testing grounds for our understanding of the chiral pion-nucleon dynamics resulting from the symmetry structure of QCD (for a recent status report, see e.g. [1]). In the absence of neutron targets, it is mandatory to consider pion production off light nuclei which also leads to the consideration of interesting aspects related to few-nucleon dynamics. In this paper, we consider pion electroproduction on the deuteron above threshold extending our previous work [2]. This is mandated by the following developments: First, the threshold results obtained in [2] can not be directly compared to the data. Furthermore, the important single scattering contribution was not calculated to fourth order (which is mandatory to describe the elementary process with sufficient accuracy) but simply shifted to its value at the photon point. Such a procedure is only well controlled for the transverse multipole. Second, coherent neutral pion production off deuterium at photon virtuality $k^{2}=-0.1 \mathrm{GeV}^{2}$ has been measured and analyzed at MAMI [3], and these data show a significant discrepancy in the dominant longitudinal cross section ( $\mathrm{S}$-wave multipole) from the prediction of [2]. In addition, new measurements of pion electroproduction off the proton at MAMI [4 at the lower photon virtuality of $k^{2}=-0.05 \mathrm{GeV}^{2}$ have led to intriguing results that can neither by explained in chiral perturbation theory nor with any sophisticated model (note the very unusual values for certain $\mathrm{P}$-waves given in that paper). Here, we want to improve the calculation for coherent pion production off the deuteron in two ways. First, in the single scattering contribution we include the full fourth order result for the transverse and longitudinal S-waves, with its parameters fixed from recent data on neutral pion photoproduction and the older NIKHEF [5] and MAMI [6] measurements for electroproduction off the proton at $k^{2}=-0.1 \mathrm{GeV}^{2}$. Note that it was recently shown that in the case of neutral pion photoproduction the fourth order corrections to the $\mathrm{P}$-wave multipoles are fairly small [7]. A similar analysis for electroproduction is not yet available, it is, however, conceivable that similar trends will persist in that case. Second, we calculate above threshold, which leads to a considerable complication in terms of the multipole expansion. While this formalism has already been developed in 8, 9, we present here a new form particularly suited for the threshold region and that most closely resembles the single nucleon multipole expansion. We restrict ourselves to $\mathrm{S}-$ and $\mathrm{P}$-waves and evaluate the three-body corrections (or meson-exchange currents) to third order in the chiral expansion. We include, however, the pion mass difference which is formally of higher order but constitutes the dominant isospin breaking effect. The single scattering part for the proton has been fixed before, and can be reliably estimated for the neutron using resonance saturation at the photon point. However, at finite photon virtuality the situation is less clear and we do not want to rely on the resonance saturation hypothesis. We therefore perform two types of fits. In the minimal fit we employ resonance saturation for a dimension four LEC (there are in principle two LECs but their sum is constrained by a low-energy theorem [10]) and use the longitudinal deuteron multipole $L_{d}$ as extracted from the MAMI data to pin down one parameter related to a particular dimension five operator [10]. In a second scenario, we do not use resonance saturation and thus have two free parameters related to the polynomial part of $L_{0+}^{n}$ which we determine from a best fit to the measured total cross sections at low pion excess energies. This still leaves sufficient predictive power since we can compare directly with the measured differential cross sections or the extracted S-wave cross section $a_{0 d}$. For doing that, one has to select a deuteron wave function. In [2], we employed the hybrid approach using various high precision wave functions together with the chirally expanded interaction kernel. Here, we also improve on that aspect using recently obtained precise effective field theory wave functions that are consistent with the power counting of the kernel [11, 12, 13].\#2 We will demonstrate that this improved calculation is in fair agreement with the MAMI deuteron data at $k^{2}=-0.1 \mathrm{GeV}^{2}$ [3], thus solving one apparent discrepancy and deepening the mystery surrounding the data of Ref. [. that a separate investigation of this second puzzle is urgently called for but should not be a topic of the present paper.

This paper is organized as follows. In Section 2 we discuss the multipole decomposition for neutral pion electroproduction off a spin-1 target. In Section 3 we briefly review the effective Lagrangian underlying the calculation and the standard power counting formulas. In Section 1 the calculation of the various contributions to the transition current (single scattering and three-body terms) is outlined. Section 5 contains the results and discussions thereof. A brief summary and outlook is given in Section 6 . The appendices include our conventions and give many more details on the calculations.

\footnotetext{
${ }^{\# 2}$ We have also performed calculations using precise phenomenological wave functions as a check. None of the results shown later depend on the choice of wave function.
} 


\section{Multipole decomposition}

The main part of this section is concerned with the multipole decomposition for the process $\gamma^{*} d \rightarrow \pi^{0} d$. To develop this, we heavily rely on the work of Arenhövel 8, 9] for the classification of the operator basis, construction of invariant amplitudes and the calculation of observables. However, in his work the main emphasis was put on the helicity basis. A formal proof of the equivalence between the multipole expansion used here and the one of Arenhövel is given in appendix A. We also summarize some basic formulae to calculate observables from the multipoles.

The invariant matrix element for the process $\gamma^{\star}(k)+d\left(p_{d}\right) \rightarrow \pi^{0}(q)+d\left(p_{d}^{\prime}\right)$, where $\gamma^{*}$ denotes the virtual photon with virtuality $k^{2} \leq 0, d$ the deuteron and $\pi^{0}$ the neutral pion with four-momentum $q_{\mu}=(\omega, \vec{q})$, can be expressed in terms of 13 invariant functions,

$$
\mathcal{M}^{\lambda}=\sum_{i=1}^{13} \mathcal{O}_{i}^{\lambda} F_{i},
$$

where the 13 operators $\mathcal{O}_{i}^{\lambda}$ are expressed in terms of combinations of the direction of the photon threemomentum $\hat{k}$, the photon polarization vector $\vec{\varepsilon}^{\lambda}$, the direction of the pion three-momentum $\hat{q}$ and the deuteron

spin vector $\vec{S}$. Here, $\lambda$ denotes the helicity of the in-coming photon, with $\lambda=0, \pm 1$. This non-relativistic form is most appropriate for near threshold production. The explicit form of the $\mathcal{O}_{i}^{\lambda}$, first written down in [8], is

$$
\begin{aligned}
\mathcal{O}_{1}^{\lambda} & =\vec{\varepsilon}^{\lambda} \cdot(\hat{k} \times \hat{q}), \quad \mathcal{O}_{2}^{\lambda}=\vec{\varepsilon}^{\lambda} \cdot(\hat{k} \times \hat{q}) \vec{S} \cdot(\hat{k} \times \hat{q}), \quad \mathcal{O}_{3}^{\lambda}=\vec{\varepsilon}^{\lambda} \cdot(\hat{k} \times(\hat{k} \times \vec{S})), \\
\mathcal{O}_{4}^{\lambda} & =\vec{\varepsilon}^{\lambda} \cdot(\hat{k} \times(\hat{q} \times \vec{S})), \quad \mathcal{O}_{5}^{\lambda}=\vec{\varepsilon}^{\lambda} \cdot(\hat{k} \times \hat{q}) \hat{k}^{[2]} \cdot S^{[2]}, \quad \mathcal{O}_{6}^{\lambda}=\vec{\varepsilon}^{\lambda} \cdot(\hat{k} \times \hat{q})[\hat{k} \times \hat{q}]^{[2]} \cdot S^{[2]}, \\
\mathcal{O}_{7}^{\lambda} & =\vec{\varepsilon}^{\lambda} \cdot(\hat{k} \times \hat{q}) \hat{q}^{[2]} \cdot S^{[2]}, \quad \mathcal{O}_{8}^{\lambda}=\vec{\varepsilon}^{\lambda} \cdot\left(\hat{k} \times\left[\hat{k} \times S^{[2]}\right]^{[1]}\right), \quad \mathcal{O}_{9}^{\lambda}=\vec{\varepsilon}^{\lambda} \cdot\left(\hat{k} \times\left[\hat{q} \times S^{[2]}\right]^{[1]}\right), \\
\mathcal{O}_{10}^{\lambda} & =\vec{\varepsilon}^{\lambda} \cdot \hat{k} \hat{k} \cdot \vec{S}, \quad \mathcal{O}_{11}^{\lambda}=\vec{\varepsilon}^{\lambda} \cdot \hat{k} \hat{q} \cdot \vec{S}, \quad \mathcal{O}_{12}^{\lambda}=\vec{\varepsilon}^{\lambda} \cdot \hat{k}[(\hat{k} \times \hat{q}) \times \hat{k}]^{[2]} \cdot S^{[2]}, \\
\mathcal{O}_{13}^{\lambda} & =\vec{\varepsilon}^{\lambda} \cdot \hat{k}[(\hat{k} \times \hat{q}) \times \hat{q}]^{[2]} \cdot S^{[2]},
\end{aligned}
$$

with

$$
\left[\vec{u} \times S^{[2]}\right]_{k}^{[1]}=u_{l} S_{l k}^{[2]}
$$

and

$$
[\vec{a} \times \vec{b}]_{i j}^{[2]}=\frac{1}{2}\left(a_{i} b_{j}+a_{j} b_{i}\right)-\frac{1}{3} \delta_{i j} \vec{a} \cdot \vec{b}
$$

the symmetric traceless tensor of second rank in standard notation. The first nine of these operators are transverse, whereas the other four are longitudinal. Note also that we work in the Coulomb gauge $\varepsilon_{0}=0$. Furthermore, the $F_{i}$ are functions of three kinematical variables, we chose here to work with the pion energy $\omega$, the photon virtuality and the scattering angle, $F_{i}=F_{i}\left(\omega, k^{2}, z\right)$. More precisely, $\theta$ is the scattering angle in the $\pi^{0} d$ center-of-mass system with $z=\cos \theta$. In what follows, we will however not display these arguments. For later use, we define the vector $\tilde{F}$ via

$$
\tilde{F}=\left(F_{1}, F_{2}, F_{3}, \ldots, F_{13}\right) .
$$

Any given tree or loop graph can now be expanded in this basis, and all observables can be expressed as functions of the $F_{i}$. Explicit expressions can be found in [9].

However, for the analysis of the data and the direct comparison with theoretical predictions, it is advantageous to use a multipole decompositon similar to the standard case of pion production off a single nucleon. The pertinent method to do that has been outlined a long time ago in [14], and we use that formalism here to develop the multipole decomposition for our case. As shown in Fig. 1, a photon with helicity $\lambda$ and multipolarity $L$ produces the neutral pion. In the final-state $\pi^{0} d$ system, the pion has relative orbital angular momentum $L_{\pi}$, which couples with the deuteron spin to the total angular momentum $J$. In the $\pi^{0} d$ frame, the invariant matrix element for the unpolarized case considered here can be written as

$$
\mathcal{M}^{\lambda}=\sum_{m, m^{\prime}}\left|m^{\prime}\right\rangle\left\langle\hat{q} m^{\prime}\left|a_{\mu}^{\lambda} J_{d}^{\mu}\left(\omega, k^{2}\right)\right| \hat{k} \lambda m\right\rangle\langle\lambda m|,
$$




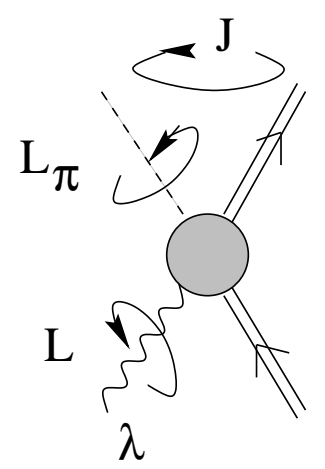

Figure 1: Graphical representation of the angular momenta involved in pion electroproduction. The in-coming photon (wiggly line) has helicity $\lambda$ and multipolarity $L$. The final $\pi^{0} d$ state is characterized by the total angular momentum $J$ and the pion (dashed line) angular momentum $L_{\pi}$. The double line denotes the deuteron.

$$
=\sum_{m, m^{\prime}}\left|m^{\prime}\right\rangle t_{m^{\prime}, \lambda, m}(\theta)\langle\lambda m|,
$$

where $a_{\mu}^{\lambda}=\varepsilon_{\mu}^{\lambda}-\left(\varepsilon_{0}^{\lambda} / k_{0}\right) k_{\mu}$ is the transverse polarisation vector and $J_{d}^{\mu}$ is the photon vector current impinging on the deuteron and we have summed over the initial and final-state deuteron magnetic quantum numbers. This matrix element can be expressed in terms of electric $E$, magnetic $M$ and longitudinal $L$ multipoles as $\$ 3$ (note that we do not give the explicit dependence on the azimuthal angle $\varphi$ here since we will only consider the transverse and the longitudinal cross section in what follows, see also the discussion below)

$$
\mathcal{M}^{\lambda}=\sum_{m, m^{\prime}} \sum_{L, L_{\pi}, J} \hat{\theta}_{m^{\prime}, m}^{L_{\pi}, \lambda} D_{\lambda, m^{\prime}, m}^{L, L_{\pi}, J} \mathcal{O}_{L_{\pi}, J}^{L, \lambda},
$$

with

$$
\begin{aligned}
\hat{\theta}_{m^{\prime}, m}^{L_{\pi}, \lambda} & =\left|m^{\prime}\right\rangle\langle m| Y_{L_{\pi}, \lambda+m-m^{\prime}}(z, \varphi=0), \\
D_{\lambda, m^{\prime}, m}^{L, L_{\pi}, J} & =\left\langle L_{\pi} \lambda+m-m^{\prime} 1 m^{\prime} \mid J \lambda+m\right\rangle\langle 1 m L \lambda \mid J \lambda+m\rangle, \\
\mathcal{O}_{L_{\pi}, J}^{L, \lambda} & =\frac{4 i \sqrt{2 \pi} \hat{L}}{\hat{J}}\left[\left(E_{L_{\pi}, J}^{L}+\lambda M_{L_{\pi}, J}^{L}\right) \delta_{|\lambda|, 1}+\frac{\sqrt{-k^{2}}}{-k_{0}} L_{L_{\pi}, J}^{L} \delta_{\lambda, 0}\right] .
\end{aligned}
$$

The first factor is proportional to the angular momentum eigenfunction in the final state (i.e. the appropriate spherical harmonics), the second term collects the pertinent Clebsch-Gordan coupling coefficients and the third term contains the dynamical information in terms of the multipoles. These multipoles depend on the pion energy and the photon virtuality. They are characterized by three labels. The superscript $L$ refers to the multipolarity while the lower indices $L_{\pi}$ and $J$ denote the orbital angular momentum and the total angular momentum of the final pion-deuteron system. We furthermore use $\hat{A}=\sqrt{2 A+1}$ for angular momentum eigenvalues. Note also that for real photons with $k^{2}=0$ (photoproduction), the photon has no longitudinal components and thus there is no coupling to the longitudinal multipoles. One can invert Eq. (2.7) and project out the multipoles, this gives

$$
\begin{aligned}
E_{L_{\pi}, J}^{L} & =\frac{1}{2}\left(1-(-)^{L_{\pi}+L}\right) \frac{\sqrt{2 \pi}}{4 i} \frac{\hat{L}}{\hat{J}} \sum_{m, m^{\prime}} D_{1, m^{\prime}, m}^{L, L_{\pi}, J} \int_{-1}^{1} d z\left\langle m^{\prime}\left|\mathcal{M}_{1}\right| m\right\rangle Y_{L_{\pi}, 1+m-m^{\prime}}(z, \varphi=0), \\
M_{L_{\pi}, J}^{L} & =\frac{1}{2}\left(1+(-)^{L_{\pi}+L}\right) \frac{\sqrt{2 \pi}}{4 i} \frac{\hat{L}}{\hat{J}} \sum_{m, m^{\prime}} D_{1, m^{\prime}, m}^{L, L_{\pi}, J} \int_{-1}^{1} d z\left\langle m^{\prime}\left|\mathcal{M}_{1}\right| m\right\rangle Y_{L_{\pi}, 1+m-m^{\prime}}(z, \varphi=0), \\
L_{L_{\pi}, J}^{L} & =\frac{1}{2}\left(1-(-)^{L_{\pi}+L}\right) \frac{-k_{0}}{\sqrt{-k^{2}}} \frac{\sqrt{2 \pi}}{4 i} \frac{\hat{L}}{\hat{J}} \sum_{m, m^{\prime}} D_{0, m^{\prime}, m}^{L, L_{m}, J} \int_{-1}^{1} d z\left\langle m^{\prime}\left|\mathcal{M}_{0}\right| m\right\rangle Y_{L_{\pi}, m-m^{\prime}}(z, \varphi=0),
\end{aligned}
$$

where due to parity, the sum $L+L_{\pi}$ has to be odd for the electric and the longitudinal multipoles and even for the magnetic ones. Since now for a given orbital angular momentum $L_{\pi}$ we have the conditions $\left|L_{\pi}-1\right| \leq J \leq L_{\pi}+1$

$\# 3$ Note the symbol $L$ is used for the multipolarity and the longitudinal multipoles. However, no confusion can arise since it is always obvious from the context what is meant. 
and $|J-1| \leq L \leq J+1$, parity allows for four different electric, four longitudinal and five magnetic multipoles, which we collect in the nine-component transverse vector $\tilde{T}_{L_{\pi}}$,

$$
\tilde{T}_{L_{\pi}}=\left(E_{L_{\pi}, L_{\pi}-1}^{L_{\pi}-1}, E_{L_{\pi}, L_{\pi}}^{L_{\pi}-1}, E_{L_{\pi}, L_{\pi}}^{L_{\pi}+1}, E_{L_{\pi}, L_{\pi}+1}^{L_{\pi}+1}, M_{L_{\pi}, L_{\pi}-1}^{L_{\pi}-2}, M_{L_{\pi}, L_{\pi}-1}^{L_{\pi}}, M_{L_{\pi}, L_{\pi}}^{L_{\pi}}, M_{L_{\pi}, L_{\pi}+1}^{L_{\pi}}, M_{L_{\pi}, L_{\pi}+1}^{L_{\pi}+2}\right),
$$

and the four-component longitudinal vector $\tilde{L}_{L_{\pi}}$,

$$
\tilde{L}_{L_{\pi}}=\left(L_{L_{\pi}, L_{\pi}-1}^{L_{\pi}-1}, L_{L_{\pi}, L_{\pi}}^{L_{\pi}-1}, L_{L_{\pi}, L_{\pi}}^{L_{\pi}+1}, L_{L_{\pi}, L_{\pi}+1}^{L_{\pi}+1}\right) .
$$

These together define the multipole vector $\tilde{\mathcal{M}}_{L_{\pi}}$,

$$
\tilde{\mathcal{M}}_{L_{\pi}}=\left(\tilde{T}_{L_{\pi}}, \tilde{L}_{L_{\pi}}\right)
$$

which has 13 components. It is straightforward albeit somewhat tedious to work out the transformation matrices between the multipole basis and the one spanned by the invariant functions $F_{i}$. The multipoles can be obtained from the $F_{i}$ by a $13 \times 13$ block-diagonal matrix, such that

$$
\tilde{\mathcal{M}}_{L_{\pi}}=\int_{-1}^{+1} d z\left(\begin{array}{cc}
D_{L_{\pi}}(z) & 0 \\
0 & E_{L_{\pi}}(z)
\end{array}\right) \tilde{F}
$$

where $D_{L_{\pi}}$ is a $9 \times 9$ and $E_{L_{\pi}}$ a $4 \times 4$ matrix. The explicit representation of these matrices in terms of Legendre polynomials is given in appendix B. Similarly, the inverse transformation is given in terms of a $9 \times 9$ matrix, called $G_{L_{\pi}}$ and a $4 \times 4$ matrix, denoted $H_{L_{\pi}}$, as

$$
\tilde{F}=\sum_{L_{\pi}=0}^{\infty}\left(\begin{array}{cc}
G_{L_{\pi}}(z) & 0 \\
0 & H_{L_{\pi}}(z)
\end{array}\right) \tilde{\mathcal{M}}_{L_{\pi}} .
$$

The explicit form of these matrices is also given in appendix B. As a non-trivial check we have shown that the product of the two $13 \times 13$ matrices in Eqs. (2.13,2.14) is indeed the unit matrix. Note that for the electric and the magnetic multipoles $L$ has to be larger or equal to one (since $|\lambda|=1$ ) and that $L_{\pi}=0$ or $L=0$ implies $J=1$. This reduces the number of allowed multipoles for $L_{\pi} \leq 2$ and leads to the lowest permissible value of $L_{\pi}$ for the various multipoles given in Table 1 .

\begin{tabular}{|l||c|c|c|c|c|}
\hline electric multipole & $E_{L_{\pi}, L_{\pi}-1}^{L_{\pi}-1}$ & $E_{L_{\pi}, L_{\pi}}^{L_{\pi}-1}$ & $E_{L_{\pi}, L_{\pi}}^{L_{\pi}+1}$ & $E_{L_{\pi}, L_{\pi}+1}^{L_{\pi}+1}$ & \\
\hline lowest value of $L_{\pi}$ & 2 & 2 & 1 & 0 & \\
\hline \hline magnetic multipole & $M_{L_{\pi}, L_{\pi}-1}^{L_{-}-2}$ & $M_{L_{\pi}, L_{\pi}-1}^{L_{\pi}}$ & $M_{L_{\pi}, L_{\pi}}^{L_{\pi}}$ & $M_{L_{\pi}, L_{\pi}+1}^{L_{\pi}}$ & $M_{L_{\pi}, L_{\pi}+1}^{L_{\pi}+2}$ \\
\hline lowest value of $L_{\pi}$ & 3 & 1 & 1 & 1 & 0 \\
\hline \hline longitudinal multipole & $L_{L_{\pi}, L_{\pi}-1}^{L_{\pi}}$ & $L_{L_{\pi}, L_{\pi}}^{L_{\pi}-1}$ & $L_{L_{\pi}, L_{\pi}}^{L_{\pi}+1}$ & $L_{L_{\pi}, L_{\pi}+1}^{L_{\pi}+1}$ & \\
\hline lowest value of $L_{\pi}$ & 2 & 1 & 1 & 0 & \\
\hline
\end{tabular}

Table 1: Lowest permissible value of $L_{\pi}$ for the various multipoles.

The unpolarized differential cross section for neutral pion electroproduction from a spin-1 target decomposes into four structure functions. However, so far no data are available for the small transverse-longitudinal and transverse-transverse interference structure functions and we thus will also not consider any azimuthal dependence here (i.e. angular dependence between the scattering and the production plane). Then, the differential cross section decomposes into a transverse and a longitudinal part, the latter being multiplied by $\varepsilon_{L}$, with $\varepsilon_{L}$ the longitudinal degree of virtual photon polarization which is related to the transverse one by

$$
\varepsilon_{L}=-\frac{k^{2}}{k_{0}^{2}} \varepsilon,
$$


with the photon energy and momentum taken in the photon-deuteron center-of-mass system. The explicit form of the transverse cross section reads

$$
\begin{aligned}
\sigma_{T} & =\frac{|\vec{q}|}{|\vec{k}|} \frac{1}{2} \sum_{\lambda= \pm 1} \frac{1}{3} \sum_{m^{\prime}, m} \int d \Omega\left|t_{m^{\prime}, \lambda, m}(\theta)\right|^{2} \\
& =4 \pi \frac{|\vec{q}|}{|\vec{k}|} \frac{8}{3} \sum_{L_{\pi}, L, J}\left\{\left|E_{L_{\pi}, J}^{L}\right|^{2}+\left|M_{L_{\pi}, J}^{L}\right|^{2}\right\} .
\end{aligned}
$$

Note that there are no electric times magnetic multipole interference terms due to the selection rules given above. Similarly, the longitudinal cross section is given entirely in terms of the longitudinal multipoles

$$
\begin{aligned}
\sigma_{L} & =\frac{|\vec{q}|}{|\vec{k}|} \frac{1}{3} \sum_{m^{\prime}, m} \int d \Omega\left|t_{m^{\prime}, 0, m}(\theta)\right|^{2} \\
& =4 \pi \frac{|\vec{q}|}{|\vec{k}|} \frac{8}{3} \frac{-k^{2}}{k_{0}^{2}} \sum_{L_{\pi}, L, J}\left|L_{L_{\pi}, J}^{L}\right|^{2} .
\end{aligned}
$$

At threshold, only the three multipoles $E_{01}^{1}, M_{01}^{2}$ and $L_{01}^{1}$ contribute and these define the transverse and longitudinal S-wave cross section $a_{0 d}$ as used in [2],

$$
a_{0 d}=\left|E_{d}\right|^{2}+\varepsilon_{L}\left|L_{d}\right|^{2},
$$

with $\left|E_{d}\right|^{2} \equiv\left|E_{01}^{1}\right|^{2}+\left|M_{01}^{2}\right|^{2}$ and $\left|L_{d}\right|^{2} \equiv\left|L_{01}^{1}\right|^{2}$. This concludes the formalism needed in this paper.

\section{Effective field theory}

In this section, we briefly discuss the effective chiral Lagrangian underlying our calculations and the corresponding power counting. For previous related work on pion photoproduction off nuclei see [15, 16].

At low energies, the relevant degrees of freedom are hadrons, in particular the Goldstone bosons linked to the spontaneous symmetry violation. We consider here the two flavor case and thus deal with the triplet of pions, collected in the matrix $U(x)$. It is straightforward to build an effective Lagrangian to describe their interactions, called $\mathcal{L}_{\pi \pi}$. This Lagrangian admits a dual expansion in small (external) momenta and quark (meson) masses as detailed below. Matter fields such as nucleons can also be included in the effective field theory based on the familiar notions of non-linearly realized chiral symmetry. These pertinent effective Lagrangian splits into two parts, $\mathcal{L}_{\pi N}$ and $\mathcal{L}_{N N}$, with the first (second) one consisting of terms with exactly one (two) nucleon(s) in the initial and the final state. Terms with more nucleon fields are of no relevance to our calculation. The pertinent contributions to neutral pion photoproduction at threshold are organized according to the standard power counting rules, which for a generic matrix element involving the interaction of any number of pions and nucleons can then be written in the form

$$
\mathcal{M}=q^{\nu} \mathcal{F}(q / \mu)
$$

where $\mu$ is a renormalization scale, and $\nu$ is a counting index, i.e. the chiral dimension of any Feynman graph. $\nu$ is, of course, intimately connected to the chiral dimension $d_{i}$ which orders the various terms in the underlying effective Lagrangian (for details, see [17]). For processes with the same number of nucleon lines in the initial and final state $(A)$, one finds 18

$$
\begin{gathered}
\nu=4-A-2 C+2 L+\sum_{i} V_{i} \Delta_{i} \\
\Delta_{i} \equiv d_{i}+n_{i} / 2-2 .
\end{gathered}
$$

where $L$ is the number of loops, $V_{i}$ is the number of vertices of type $i, d_{i}$ is the number of derivatives or powers of $M_{\pi}$ which contribute to an interaction of type $i$ with $n_{i}$ nucleon fields, and $C$ is the number of separately connected pieces. This formula is important because chiral symmetry places a lower bound: $\Delta_{i} \geq 0$. Hence the leading irreducible graphs are tree graphs $(L=0)$ with the maximum number $C$ of separately connected 
pieces, constructed from vertices with $\Delta_{i}=0$. In the presence of an electromagnetic field, this formula is slightly modified. Photons couple via the electromagnetic field strength tensor and by minimal substitution. This has the simple effect of modifying the lower bound on $\Delta_{i}$ to $\Delta_{i} \geq-1$, and of introducing an expansion in the electromagnetic coupling, $e$. Throughout, we work to first order in $e$, with one exception to be discussed below. For more details on the counting, we refer to [15]. In what follows, we will work within the oneloop approximation to order $q^{3}$ (notice that we refer here to the chiral dimension used to organize the various terms in the calculation of the single-nucleon photoproduction amplitudes), with the exception of the S-wave contribution to the elementary process $\gamma^{\star} N \rightarrow \pi^{0} N$ (as discussed in the introduction). In terms of the counting index $\nu$, we include all terms with $\nu=4-3 A=-2$ and $\nu=5-3 A=-1$. Consequently, the effective Lagrangian consists of the following pieces:

$$
\mathcal{L}_{\text {eff }}=\mathcal{L}_{\pi \pi}^{(2)}+\mathcal{L}_{\pi N}^{(1)}+\mathcal{L}_{\pi N}^{(2)}+\mathcal{L}_{\pi N}^{(3)}\left[+\mathcal{L}_{\pi N}^{(4)}\right]+\mathcal{L}_{N N}^{(0)}+\mathcal{L}_{N N}^{(2)}
$$

where the index $(i)$ gives the chiral dimension $d_{i}$ (number of derivative and/or meson mass insertions). The form of $\mathcal{L}_{\pi \pi}^{(2)}+\mathcal{L}_{\pi N}^{(1)}$ is standard. The terms from $\mathcal{L}_{\pi N}^{(3)}+\mathcal{L}_{\pi N}^{(4)}$ contributing to the single-nucleon electroproduction amplitudes are given in Ref. 10]. Note that the square brackets in Eq. (3.3) indicate that such fourth order terms are only taken for the S-wave single nucleon production amplitudes ${ }^{\# 4}$. The effective Lagrangian can also be used to generate deuteron wave functions of sufficient precision, as done in Refs. 12, 13] based on a modified Weinberg power counting. We use the NLO and the node-less NNLO* wave functions from [13] for the allowed cut-off range $\Lambda=500 \ldots 600 \mathrm{MeV}$, where the cut-off stems from the regulator function in the LippmannSchwinger equation used to generate the bound and the scattering states (for a more detailed discussion giving also the explicit form of $\mathcal{L}_{N N}^{(0,2)}$, see e.g. Ref. 11]). As a check, we have also made use of the hybrid approach of [19], sewing precise phenomenological wave functions to the chirally expanded kernel. None of the results discussed later depend on the choice of wave function and we therefore will only present numbers for the chiral EFT wave functions. After these general remarks, let us now turn to the actual calculations.

\section{Anatomy of the calculation}

In this section, we outline how the various contributions to the multipoles and the observables are calculated. First, we briefly discuss the separation of the transition matrix elements into two- and three-body terms (or, in nuclear physics language, impulse and meson-exchange terms). Then, these two types of contributions are discussed separately, in particular we stress the differences to the threshold calculation of [2]. Many details are relegated to the appendices.

\subsection{General remarks}

Consider first a generic diagram for neutral pion electroproduction off the deuteron as shown in Fig. 2. The interaction kernel decomposes into two distinct and different pieces. First, the virtual photon can produce the pion on either the proton of the neutron, with the other nucleon acting as a mere spectator. This is called the single scattering contribution (ss), compare Fig. 2. It is important to note that to properly account for this one has to transform from the photon-nucleon to the photon-deuteron center-of-mass system as discussed below. Second, all other terms in the interaction kernel involve both nucleons, comprising the so-called three-body (tb) interactions (see again Fig. 2). To be specific, the transition matrix element for pion production of the deuteron has the form

$$
\mathcal{M}^{\lambda}=\sum_{m_{s}, m_{s}^{\prime}}\left|m_{s}^{\prime}\right\rangle\left\langle\vec{p}_{d}^{\prime} \vec{q}\left|a_{\mu}^{\lambda} J_{d}^{\mu}\right| \vec{p}_{d} \vec{k}\right\rangle\left\langle m_{s}\right|
$$

in terms of the three-momenta of the in-coming (out-going) deuteron, $\vec{p}_{d}$ and $\vec{p}_{d}{ }^{\prime}$, respectively. Here, $J_{d}^{\mu}$ denotes the vector current impinging on the deuteron. To unravel the underlying structure of the deuteron, one expresses this matrix element in terms of the two-nucleon current, $J_{\mathrm{NN}}$. This current then separates into the two terms just discussed,

$$
\left\langle\vec{p}_{d}^{\prime} \vec{q}\left|a_{\mu}^{\lambda} J_{d}^{\mu}\right| \vec{p}_{d} \vec{k}\right\rangle=\frac{1}{(2 \pi)^{3}} \sqrt{\frac{E_{d} E_{d}^{\prime}}{4 E_{1} E_{1}^{\prime} E_{2} E_{2}^{\prime}}}\left\langle\vec{p}_{d}^{\prime} \vec{q}\left|a_{\mu}^{\lambda} J_{N N}^{\mu}\right| \vec{p}_{d} \vec{k}\right\rangle
$$

\footnotetext{
\#4 In fact, one also has to account for one particular dimension five operator as explained in [10, see also Sect. 4.2.
} 


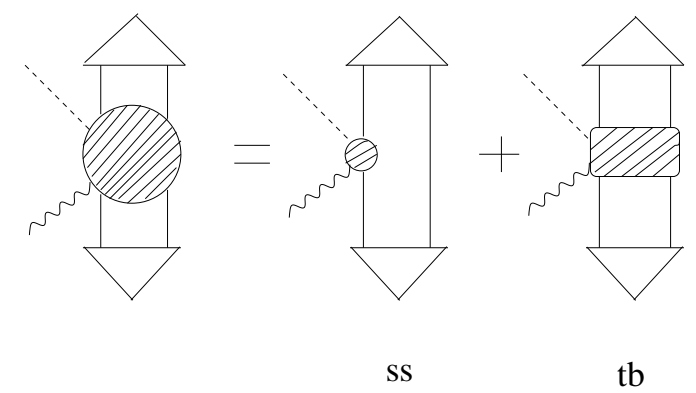

Figure 2: Decomposition of the full interaction kernel (as shown in leftmost diagram by the shaded circle) into the single scattering (ss) and the three-body (tb) contribution. The triangle symbolizes the deuteron wave function.

$$
=\frac{1}{(2 \pi)^{3}} \sqrt{\frac{E_{d} E_{d}^{\prime}}{4 E_{1} E_{1}^{\prime} E_{2} E_{2}^{\prime}}}\left(\left\langle\vec{p}_{d}^{\prime} \vec{q}\left|a_{\mu}^{\lambda} J_{\mathrm{NN}}^{\mu}\right| \vec{p}_{d} \vec{k}\right\rangle^{\mathrm{ss}}+\left\langle\vec{p}_{d}^{\prime} \vec{q}\left|a_{\mu}^{\lambda} J_{\mathrm{NN}}^{\mu}\right| \vec{p}_{d} \vec{k}\right\rangle^{\mathrm{tb}}\right)
$$

where $E_{i}\left(E_{i}^{\prime}\right)$ denotes the energy of nucleon $i(i=1,2)$ in the initial (final) state. In what follows, we will work in the approximation that pion is produced in $\mathrm{S}-$ or $\mathrm{P}$-waves only, that is we allow for $L_{\pi}=0,1$. This means that we have to consider three $\mathrm{S}$-wave $\left(E_{01}^{1}, L_{01}^{1}, M_{01}^{2}\right)$ and eight $\mathrm{P}$-wave multipoles $\left(E_{11}^{2}, E_{12}^{2}, M_{10}^{1}, M_{11}^{1}, M_{12}^{1}, M_{12}^{3}, L_{11}^{0}\right.$, $\left.L_{11}^{2}, L_{12}^{2}\right)$, compare Table 1. Only if one allows for higher partial waves, all thirteen different structures discussed in Section 2 will contribute. We will now discuss the single scattering and the three-body contributions in more detail.

\subsection{Single scattering contribution}

In the previous paragraph, we have introduced the single scattering contribution to the two-nucleon current. It has the following generic form

$$
a_{\mu}^{\lambda} J_{\mathrm{NN}}^{\mathrm{ss}, \mu}=\frac{1}{2}\left(2 m(2 \pi)^{3} \delta\left(\vec{p}_{1}^{\prime}-\vec{p}_{1}\right)\left[a_{\mu}^{\lambda} J^{\pi^{0} p, \mu}+a_{\mu}^{\lambda} J^{\pi^{0} n, \mu}\right]_{2}+(1 \leftrightarrow 2)\right)
$$

with $\vec{p}_{1}-\vec{p}_{1}{ }^{\prime}=\vec{p}-\vec{p}^{\prime}-\vec{k} / 2+\vec{q} / 2$ in terms of the in-coming (out-going) relative nucleon cms momenta $\vec{p}\left(\vec{p}^{\prime}\right)$, the pion and the photon momenta and similarly for $\vec{p}_{2}-\vec{p}_{2}{ }^{\prime}$. The isoscalar current is then expressed in terms of the conventional CGLN [20] amplitudes,

$$
\left[a_{\mu}^{\lambda} J^{\pi^{0} p, \mu}+a_{\mu}^{\lambda} J^{\pi^{0} n, \mu}\right]_{i}=8 \pi W_{i}^{\star} \sum_{L_{\pi}=0}^{\infty} \tilde{O}_{i}^{\mathrm{ss}}\left(\hat{k}^{\star}, \hat{q}^{\star}\right) \cdot\left(\begin{array}{cc}
G_{L_{\pi}}^{\mathrm{ss}}\left(z^{\star}\right) & 0 \\
0 & H_{L_{\pi}}^{\mathrm{ss}}\left(z^{\star}\right)
\end{array}\right) \cdot\left(\tilde{M}_{L_{\pi}^{\mathrm{ss}, \pi^{0} p}+\tilde{M}_{L_{\pi}}^{\mathrm{ss}, \pi^{0} n}}\right)
$$

where the stared quantities refer to the center-of-mass system of nucleon $i$, the $\tilde{M}_{L_{\pi}}^{\text {ss }}$ denote the single nucleon multipoles, the explicit form of the operators $\tilde{O}_{i}^{\mathrm{ss}}$ is given in appendix $囚$ and the corresponding transformation matrix for the transverse and longitudinal multipoles is standard [21]. Of course, one has to transform these expressions from the pion-nucleon to the pion-deuteron center-of-mass system. This is described in some detail in appendix 9. When sandwiched between the deuteron wave functions, one has to deal with integrals of the type

$$
\int d^{3} p \phi^{*}\left(\vec{p}-\frac{\vec{k}}{2}+\frac{\vec{q}}{2}\right)\left(\mathcal{O}_{1}+\overrightarrow{\mathcal{O}}_{2} \cdot \vec{p}\right) \phi(\vec{p})
$$

where $\phi(p)$ denotes the momentum space deuteron wave function, and $\mathcal{O}_{1}, \overrightarrow{\mathcal{O}}_{2}$ are arbitrary spin structures. To work in coordinate space, one has to Fourier transform these expressions, using the basic integrals

$$
\int d^{3} p \phi^{*}\left(\vec{p}-\frac{\vec{k}}{2}+\frac{\vec{q}}{2}\right) \mathcal{O}_{1} \phi(\vec{p})=\int d^{3} r \phi^{\dagger}(\vec{r}) \mathcal{O}_{1} \phi(\vec{r}) \mathrm{e}^{-i(\vec{k}-\vec{q}) \cdot \vec{r} / 2}
$$




$$
\int d^{3} p \phi^{*}\left(\vec{p}-\frac{\vec{k}}{2}+\frac{\vec{q}}{2}\right) \mathcal{O}_{2, i} p_{i} \phi(\vec{p})=\int d^{3} r \phi^{\dagger}(\vec{r}) \mathcal{O}_{2, i} \frac{1}{i} \partial_{r i} \phi(\vec{r}) \mathrm{e}^{-i(\vec{k}-\vec{q}) \cdot \vec{r} / 2},
$$

and further decomposing the deuteron co-ordinate space wave function $\phi(\vec{r})$ into its radial $\mathrm{S}^{-}$and $\mathrm{D}$-wave components,

$$
\phi(r)=\frac{1}{\sqrt{4 \pi}}\left(\frac{u(r)}{r}+\sqrt{\frac{1}{8}} \frac{w(r)}{r} S_{12}(\hat{r})\right) .
$$

Here, $S_{12}(\hat{r})$ is the usual second order tensor operator. We also remark that in [2] we had expressed the overlap integrals in a factorized form, more specifically, the transverse and longitudinal deuteron multipoles could be written as products of the single nucleon S-wave multipoles and a set of deuteron form factors. Such a procedure becomes very complicated above threshold and is not transparent, therefore we do not follow such a path here in detail (although we have performed some calculations in that framework to have a further check on the numerics). Still, it is important to stress that the single scattering contribution is strongly suppressed with increasing photon virtuality because of the decreasing overlap integrals in Eq. (4.5).

Again, we work in the $\mathrm{S}-$ and $\mathrm{P}$-wave approximation. The corresponding single nucleon multipoles are subject to the chiral expansion. We work to first non-trivial loop order, i.e. to third order, with the exception of the proton and neutron S-wave multipoles $E_{0+}^{n, p}$ and $L_{0+}^{n, p}$. We take the form given in [10] which includes all fourth order terms and one particular fifth order term necessary to separate cleanly the longitudinal from the transverse piece. We refer to that paper for the explicit expressions of the single nucleon transition current. Here, we only spell out the generic form for the S-waves,

$$
\mathcal{S}=\mathcal{S}^{\text {Born }}+\mathcal{S}^{\mathrm{q}^{3}-\text { loop }}+\mathcal{S}^{\mathrm{q}^{4}-\text { loop }}+\mathcal{S}^{\mathrm{ct}},
$$

with $\mathcal{S}=E_{0+}^{p, n}$ or $L_{0+}^{p, n}$. At fourth order one has two local operators $\sim k^{2}$ with the LECs $a_{3}^{I}, a_{4}^{I}(I=p, n)$. However, a particular low-energy theorem (LET) strongly correlates these two LECs, in the soft-pion limit one has $a_{3}^{I}+a_{4}^{I}=0$. To break this correlation that is not observed in the proton data, one has to include a correction to the LET of the form $L_{0+}^{\text {ct,I }}=-e M_{\pi}^{2} k^{2} a_{5}^{I}$ which is formally of fifth chiral order (in the effective Lagrangian). As noted before, with that we are able to describe the proton data of [5. 6] for $\gamma^{\star} p \rightarrow \pi^{0} p$ at $k^{2}=-0.1 \mathrm{GeV}^{2}$ but not the more recent data of [4] at half the photon virtuality (as discussed in the introduction). For the LECs related to the neutron amplitude, we follow two strategies. First, we fix $a_{3}^{n}=a_{4}^{n}$ from resonance saturation as detailed in Ref. 10] and determine $a_{5}^{n}$ from a fit to the empirical threshold amplitude $L_{d}$ of Ref. [3]. Second, we leave both $a_{3}^{n}$ and $a_{4}^{n}$ as free parameters thus relaxing the constraint due to the LET and fit to the threshold total cross sections of Ref. [3]. In what follows, we will call these two procedures fit 1 and fit 2, respectively. The scaling procedure performed in [2] was done too simplistically for the longitudinal S-wave multipole leading to the too large S-wave cross section.

There is one additional point that deserves particular discussion. With increasing photon excess energy (that is the energy normalized to the threshold energy), one should observe two unitary cusps due to the opening of the $\pi^{+} n n$ and the $\pi^{-} p p$ channel for neutral pion production off the proton and the neutron, respectively. Indeed, if one does not boost the energy dependent multipoles as suggested in [22], two cusps are visible as shown in Fig. 3 for the real part of $E_{10}^{1}$ together with the corresponding growth of the imaginary part $\$ 5$. The effect of applying the full boost correction, i.e. also changing the arguments of the multipole amplitudes, turns out to be small, as also shown in Fig. 3. The real part is shifted by a few percent and the imaginary part is almost unaffected for the pion energies considered here. Note that in all non-analytic terms, like e.g. the energy dependence of the $\mathrm{S}$-waves $\sim \sqrt{1-\omega^{2} / \omega_{c}^{2}}$ (and similar terms in the $\mathrm{P}$-waves), we always use the physical values for the $n n \pi^{+}$ and $p p \pi^{-}$thresholds given by $\omega_{c}$. This is consistent with the chiral expansion and the analytic structure of the amplitudes.

\subsection{Three-body contribution}

We now turn to the three-body contribution. Above threshold, we have 8 diagrams contributing at third order, see Fig. 6 . Note that in contrast to earlier works, we differentiate between the charged and neutral pion masses

\footnotetext{
\#5 Note that we work with $M_{\pi^{0}}=134.97 \mathrm{MeV}$ and $M_{\pi^{+}}=140.13 \mathrm{MeV}$ or $M_{\pi^{+}}=142.53 \mathrm{MeV}$, to account for the neutron-proton mass difference in the rescattering diagrams. A detailed discussion of this point is given in Ref. 23.
} 


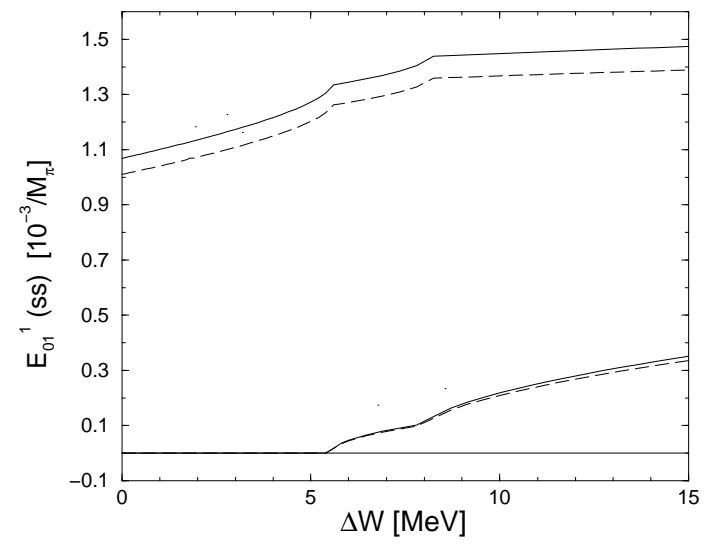

Figure 3: Effect of the Lorentz boost on the arguments of the multipoles. Shown are the real (upper two lines) and the imaginary part (lower two lines) of the single scattering contribution to the electric multipole $E_{01}^{1}$ for varying pion excess energy $\Delta W$ at fixed photon virtuality $k^{2}=$ $-0.1 \mathrm{GeV}^{2}$. Solid (dot-dashed) lines: with (without) boost.

also in these graphs, although this is formally an effect of higher order. We remark that one can combine these various contributions into two distinct classes with one and two pion propagators, respectively, the so-called rescattering and pion-in-flight diagrams.

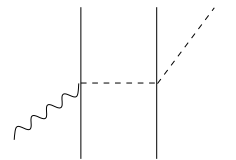

a)

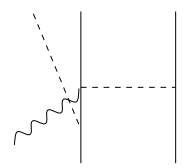

e)

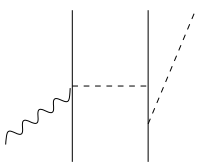

b)

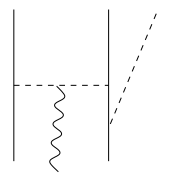

f)

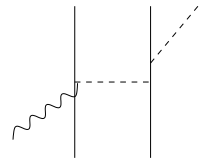

c)

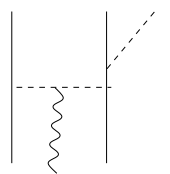

g)

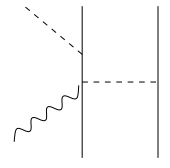

d)

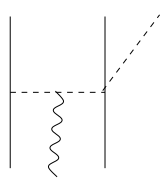

h)

Figure 4: Three-body interactions which contribute to neutral pion electroproduction at threshold to order $q^{3}$ (in the Coulomb gauge). Here, solid, dashed and wiggly lines denote nucleons, pions and photons, in order.

The explicit analytical expressions for the corresponding matrix elements in momentum space are:

Diagrams a) + b) $+\mathrm{c})+\mathrm{d})+\mathrm{e})($ rescattering type)

$$
\mathcal{M}_{a+b+c+d+e}=2 \frac{e g_{A} m^{2}}{F_{\pi}^{3}}\left(\vec{\varepsilon} \cdot \vec{S}\left[g_{A}^{2} \frac{1}{q_{0}} \vec{q} \cdot \vec{q}^{\prime}-q_{0}\right]-g_{A}^{2} \frac{1}{q_{0}} \vec{S} \cdot \vec{q}^{\prime} \vec{\varepsilon} \cdot \vec{q}\right) \frac{1}{\vec{q}^{\prime 2}+\delta^{2}}\left[\vec{\tau}_{1} \cdot \vec{\tau}_{2}-\tau_{1}^{3} \tau_{2}^{3}\right] .
$$

Here, $q^{\prime}=\left(q_{0}^{\prime}, \vec{q}^{\prime}\right)$ with $q_{0}^{\prime}=q_{0}+\mathcal{O}(1 / m)$ is the four-momentum of the exchanged pion and

$$
\delta^{2}=M_{\pi^{+}}^{2}-q_{0}^{2}-i \epsilon .
$$


$\underline{\text { Diagrams } \mathrm{f})+\mathrm{g})+\mathrm{h})}($ pion-in-flight type)

$$
\mathcal{M}_{f+g+h}=-2 \frac{e g_{A} m^{2}}{F_{\pi}^{3}} \vec{\varepsilon} \cdot\left(\vec{q}^{\prime \prime}+\vec{q}^{\prime}\right) \vec{S} \cdot \vec{q}^{\prime \prime} \frac{g_{A}^{2} q_{0}^{-1} \vec{q} \cdot \vec{q}^{\prime}-q_{0}}{\left(\vec{q}^{\prime \prime} 2+M_{\pi^{+}}^{2}\right)\left(\vec{q}^{\prime 2}+\delta^{2}\right)}\left[\vec{\tau}_{1} \cdot \vec{\tau}_{2}-\tau_{1}^{3} \tau_{2}^{3}\right] .
$$

Here, we use the following convention. The intermediate pion has momentum $\vec{q}^{\prime \prime}$ after emission from the left nucleon line and before the interaction with the photon. After that, the momentum is $\vec{q}^{\prime}$ and the pion is absorbed on the right nucleon. Note that for both classes of diagrams, the factor 2 in front takes care of the interchange $1 \leftrightarrow 2$. We remark that in contrast to previous work [2, 16] we differentiate between the charged and neutral pion masses for the exchanged meson. While that is formally an effect of higher order, we still consider it here because it was already shown to be the dominant isospin breaking effect in the investigation of pion photoproduction off nucleons, first discussed in the context of chiral perturbation theory in [24]. In essence, we have calculated all three-body graphs for the two different values of $\omega_{c}$ corresponding to the opening of the $p p \pi^{-}$and the $n n \pi^{+}$channels and performed the necessary average. Since these operators are sandwiched between the deuteron wave functions and one has to integrate over all momenta, one picks up an imaginary part from the intermediate $N N \pi$ state, i.e. the corresponding propagators have to be split into a real (principal value) and an imaginary part. It appears when the momentum of the exchanged pion is equal to the charged pion mass, that is at an excess energy of

$$
\Delta W_{c}=\omega_{c}+\sqrt{m_{d}^{2}+\omega_{c}^{2}-M_{\pi^{0}}^{2}}-W_{0}=5.3(7.9) \mathrm{MeV},
$$

for the $p p \pi^{-}\left(n n \pi^{+}\right)$intermediate state. Here, $W_{0}=m_{d}+M_{\pi^{0}}$ is the threshold energy. These poles will reveal itself as a cusp-like structure in the corresponding multipoles (if one considers the three-body terms separately, see Section 5). As before, we have restricted the calculation to relative $\mathrm{S}-$ and $\mathrm{P}$-waves. We have also performed calculations without this truncation, which gives a relative measure of the contribution from D- (and higher partial) waves. More specifically, consider a typical coordinate space integral,

$$
\int d \Omega_{r} \mathrm{e}^{-i(\vec{k} x+\vec{q} / 2) \cdot \vec{r}}=4 \pi j_{0}(a r)=4 \pi \sum_{L=0}^{\infty}(-\operatorname{sgn}(x))^{L}(2 L+1) j_{L}(b) j_{L}(c) P_{L}(\hat{q} \cdot \hat{k}),
$$

with $a=|\vec{k} x+\vec{q} / 2| r, b=k r|x|$ and $c=q r / 2$. Note that the coefficient $a$ in the Bessel function $j_{0}(a r)$ contains the explicit angular dependence. In case of the $\mathrm{S}-$ and $\mathrm{P}$-wave approximation, one operates with the projection matrix on the full sum and the series in Eq. (4.13) truncates after the first few terms. We have found that these differences are very small, justifying a posteriori the assumption of only retaining the $\mathrm{S}-$ and $\mathrm{P}$-waves.

\section{$5 \quad$ Results and discussion}

In this section, we display the results for the multipoles, differential and total cross sections and the S-wave cross section $a_{0 d}$ for the two fit strategies. We have performed calculations with the chiral EFT wave functions at NLO [11] and NNLO* 12, 13] for cut-offs in the range of 500 to $600 \mathrm{MeV}$. Since the results for the observables are very similar for all these various wave functions, we only show these for the NNLO* wave function with $\Lambda=600 \mathrm{MeV}$. The fitted LECs vary mildly for the various wave functions as shown in Table 2. We note that all these LECs are of natural size (note that the value for $a_{5}^{n}$ appears unnaturally large due to the particular definition of this LEC as used in Ref.[10], see also the discussion in that paper). We remark that the results using the Bonn wave function as employed in Ref. [2] are fully consistent with the ones based on the chiral EFT wave functions and we thus refrain from displaying these numbers here.

The real parts of the various $\mathrm{S}-$ and $\mathrm{P}$-wave multipoles are displayed in Figures $5 \mathrm{H} 8$ by the solid lines for fit 2 using the NNLO* wave functions with $\Lambda=600 \mathrm{MeV}$. The corresponding single scattering contribution is also shown (dashed lines) \#6. As found in previous calculations, the three-body effects are sizeable, especially in the S-waves. In contrast to previous attempts using meson-exchange models this does not pose a problem for extracting the single scattering contribution because one can systematically calculate the higher order corrections

\footnotetext{
${ }^{\# 6}$ We refrain from showing these multipoles using the other wave functions for fit 2 because they come out very similar. There are some differences in the multipoles for fit 1 , as will be discussed for the $\mathrm{S}$-waves later on.
} 

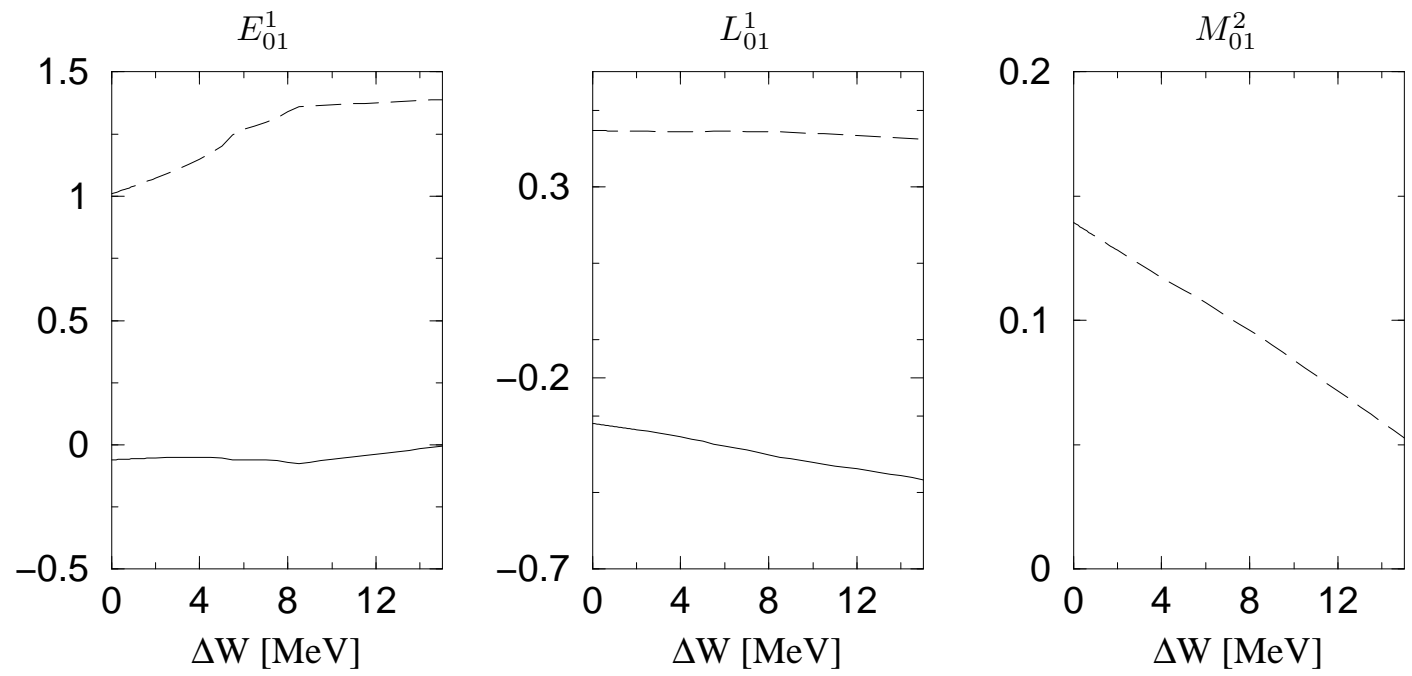

Figure 5: S-wave multipoles as a function of the pion excess energy $\Delta W$ for photon virtuality $k^{2}=-0.1 \mathrm{GeV}^{2}$. The solid (dashed) line shows the total (single scattering) contribution. Note the absence of cusp effects in the full result for $E_{01}^{1}$. For $M_{01}^{2}$, there is no tb contribution to this order in the chiral expansion. Units are $10^{-3} / M_{\pi^{+}}$.
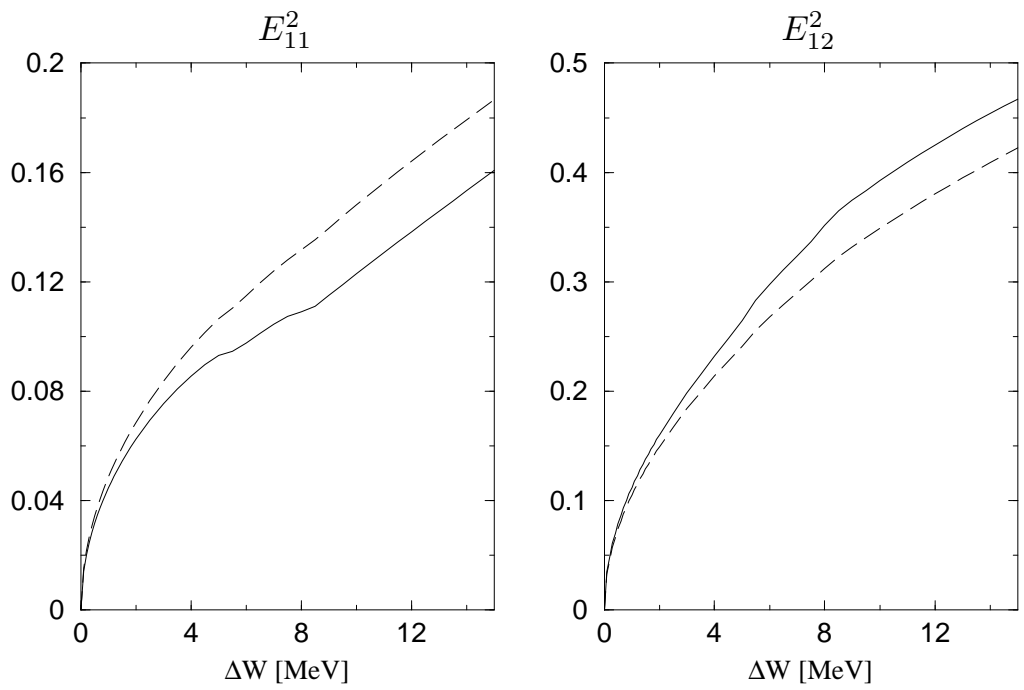

Figure 6: Electric $\mathrm{P}$-wave multipoles as a function of the pion excess energy $\Delta W$ for photon virtuality $k^{2}=-0.1 \mathrm{GeV}^{2}$. The solid (dashed) line shows the total (single scattering) contribution. Units are $10^{-3} / M_{\pi^{+}}$. 

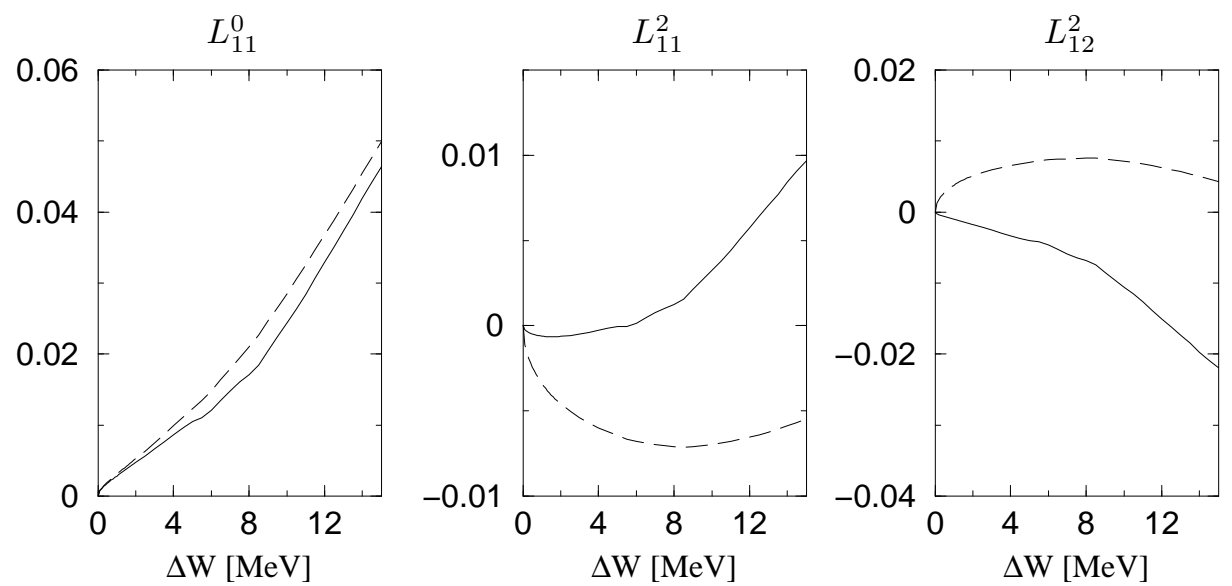

Figure 7: Longitudinal $\mathrm{P}$-wave multipoles as a function of the pion excess energy $\Delta W$ for photon virtuality $k^{2}=-0.1 \mathrm{GeV}^{2}$. The solid (dashed) line shows the total (single scattering) contribution. Units are $10^{-3} / M_{\pi^{+}}$.
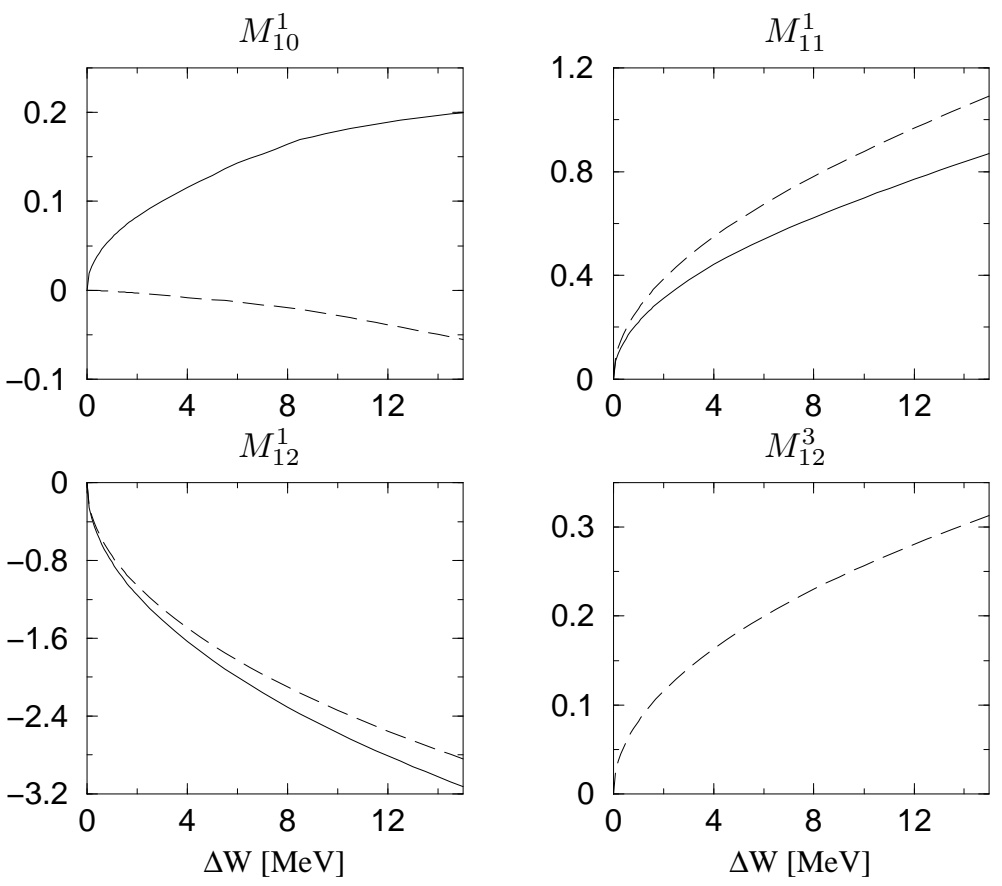

Figure 8: Magnetic $\mathrm{P}$-wave multipoles as a function of the pion excess energy $\Delta W$ for photon virtuality $k^{2}=-0.1 \mathrm{GeV}^{2}$. The solid (dashed) line shows the total (single scattering) contribution. Units are $10^{-3} / M_{\pi^{+}}$. 


\begin{tabular}{|c||c|c|c|c|}
\hline w.f. & NLO-500 & NLO-600 & NNLO*-500 & NNLO*-600 \\
\hline$a_{3}^{n}\left[\mathrm{GeV}^{-4}\right]$ & 4.010 & 3.459 & 4.832 & 4.966 \\
$a_{4}^{n}\left[\mathrm{GeV}^{-4}\right]$ & -5.925 & -5.745 & -5.895 & -5.660 \\
\hline$a_{5}^{n}\left[\mathrm{GeV}^{-5}\right]$ & -34.49 & -36.45 & -29.86 & -27.51 \\
\hline
\end{tabular}

Table 2: Values of the fitted LECs for the various wave functions (w.f.). The values for $a_{3,4}^{n}$ refer to the fits 2 , whereas the corresponding $a_{5}^{n}$ belongs to the respective fits 1.

to the three-body terms. This was indeed done for the case of neutral pion photoproduction off deuterium in [16] and we anticipate a similar result for the case under consideration (a complete fourth order calculation for the deuteron case can only be done when a similar investigation for single nucleon electroproduction is available and the already discussed inconsistencies have been resolved). We note in particular the cusp-like effects in certain $\mathrm{P}$-wave multipoles due to the pion mass difference in the three-body contributions as discussed in Section 4.3 . A comparable multipole analysis of the data is not yet available. The multipoles extracted in Ref. [3] are based on the simplifying assumptions of constant $\mathrm{S}$-waves and $\mathrm{P}$-waves that solely depend on the pion center-of-mass momentum. A direct comparison of the multipoles obtained here with the ones of [3] have therefore to be taken cum grano salis. Nonetheless we have performed the fits of type 1 by matching to the empirical value of $\left|L_{d}\right|$ to get a better handle on the theoretical uncertainties of our calculation. Note also that the recently proposed exact cancellation 25] between the single nucleon rescattering and the charge exchange three-body diagram at threshold is visible in the S-wave multipoles, the cusp effects from the ss and tb terms neatly cancel, cf. Fig. 5. However, this argument only affects a subset of graphs and does not lead to the conclusion that one is essentially sensitive to the single scattering amplitude, as reflected in our results.

In Figs. 9,10 we show the differential cross sections for fits 1 and 2 employing the NNLO*-600 wave functions in comparison to the MAMI data [3]. These two lines corresponding to the two fit procedures can be considered as a measure of the theoretical uncertainty at this order. This uncertainty is comparable to the the experimental errors. The bell-shape behaviour of the differential cross sections at the higher values of the pion excess energy is similar to what is found in neutral pion photoproduction off protons and can be traced back to the large and delta-dominated third order $\mathrm{P}$-wave LECs $b_{p, n}$, which are well described in terms of resonance saturation. Within large fluctuations, the data show more of a backward-forward angle asymmetry. This feature might be better described when the P-waves have also been worked out to forth order, but it is fair to state that we do not find sizeable discrepancies between the data and the theoretical predictions. As in pion production off the proton, the $\mathrm{S}$-wave multipoles are only dominant very close to threshold and the $\mathrm{P}$-waves start to dominate at excess energies of a few $\mathrm{MeV}$.

The corresponding total cross sections as a function of the excess energy $\Delta W$ and of the photon polarization $\varepsilon$ are shown in Figs. 11 and 12 for fit 1 and in Figs. 13 and 14 for fit 2 and the NNLO*-600 wave function, respectively. We notice that for fit 1 with increasing excess energy and, in particular, with increasing photon polarization the data are systematically below the chiral prediction. Due to the fitting procedure, the slopes of the various curves for the Rosenbluth separation shown in Figs. 12 are of course correct. On the other hand, the fitting procedure 2 gives an overall better description of the total cross sections, cf. Fig. 13 with a somewhat too small longitudinal S-wave contribution, as most clearly seen in Fig. 14, where again the Rosenbluth separation of the total cross section is plotted. These observation can further be sharpened by considering the dominant longitudinal multipoles as visualized in Fig. 15, where the transverse and longitudinal threshold S-wave multipoles $E_{d}$ and $L_{d}$ are shown in comparison to the data for fits 1 and 2 using the NNLO*600 wave function. We also note that $E_{d}$ is slightly below the data from SAL 26, whereas the prediction from [16] was by the same amount above the data. This can be traced back to a variety of effects. First, we use slightly different input parameters (for the neutron) so that the single scattering contribution is somewhat reduced. Further, in contrast to Ref. [16] we include the pion mass difference in the three-body contribution, which further reduces $E_{d}$ by about $0.3 \cdot 10^{-3} / M_{\pi}$, compare Fig. 目 (the energy dependence of $E_{01}^{1}$ is almost the same at the photon point $k^{2}=0$ ). Also, our treatment of the Fermi motion (boost correction) is improved as compared to that paper and we thus have an additional reduction of $E_{d}$. Given that there are other fourth order 

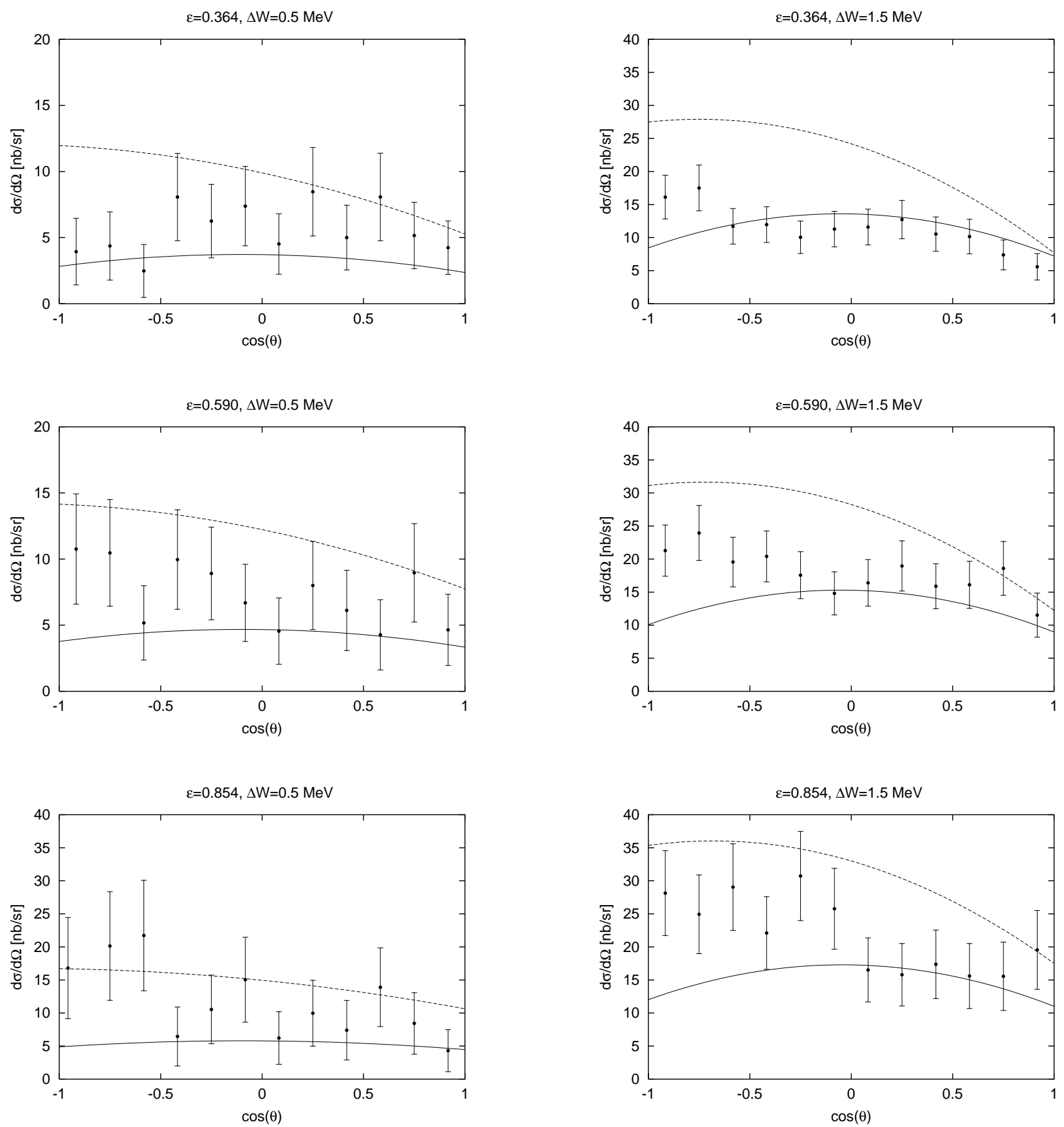

Figure 9: Differential cross section at $\Delta W=0.5 \mathrm{MeV}$ (left column) and $\Delta W=1.5 \mathrm{MeV}$ (right column) at three different values of the photon polarization for the $\mathrm{NNLO}^{*}-600$ wave function in comparison to the MAMI data [3]. Fit 1 (2): dashed (solid) lines. 

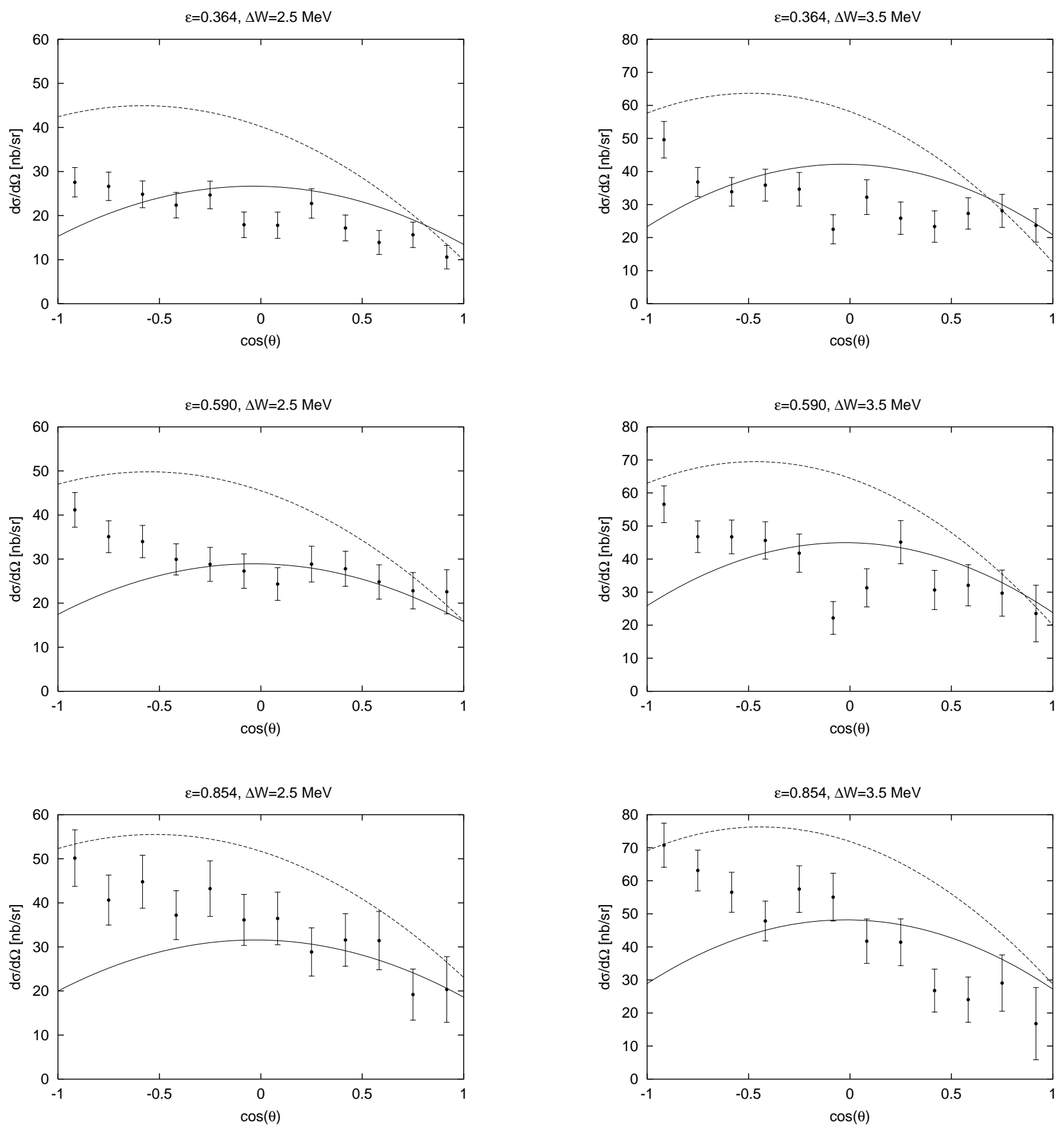

Figure 10: Differential cross section at $\Delta W=2.5 \mathrm{MeV}$ (left column) and $\Delta W=3.5 \mathrm{MeV}$ (right column) at three different values of the photon polarization for the $\mathrm{NNLO}^{*}-600$ wave function in comparison to the MAMI data [3]. Fit 1 (2): dashed (solid) lines. 


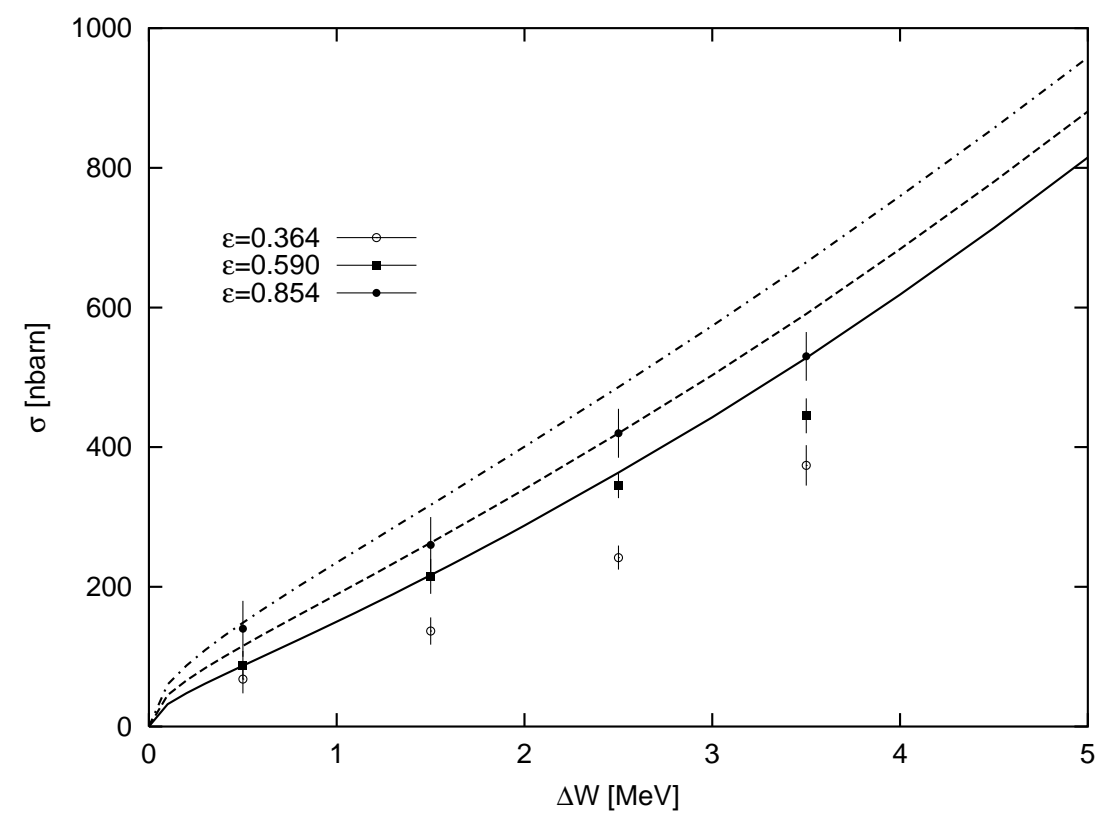

Figure 11: Total cross section as a function of $\Delta W$ for three different values of the photon polarization in comparison to the MAMI data [3] for fit 1 and the NNLO*-600 wave function.

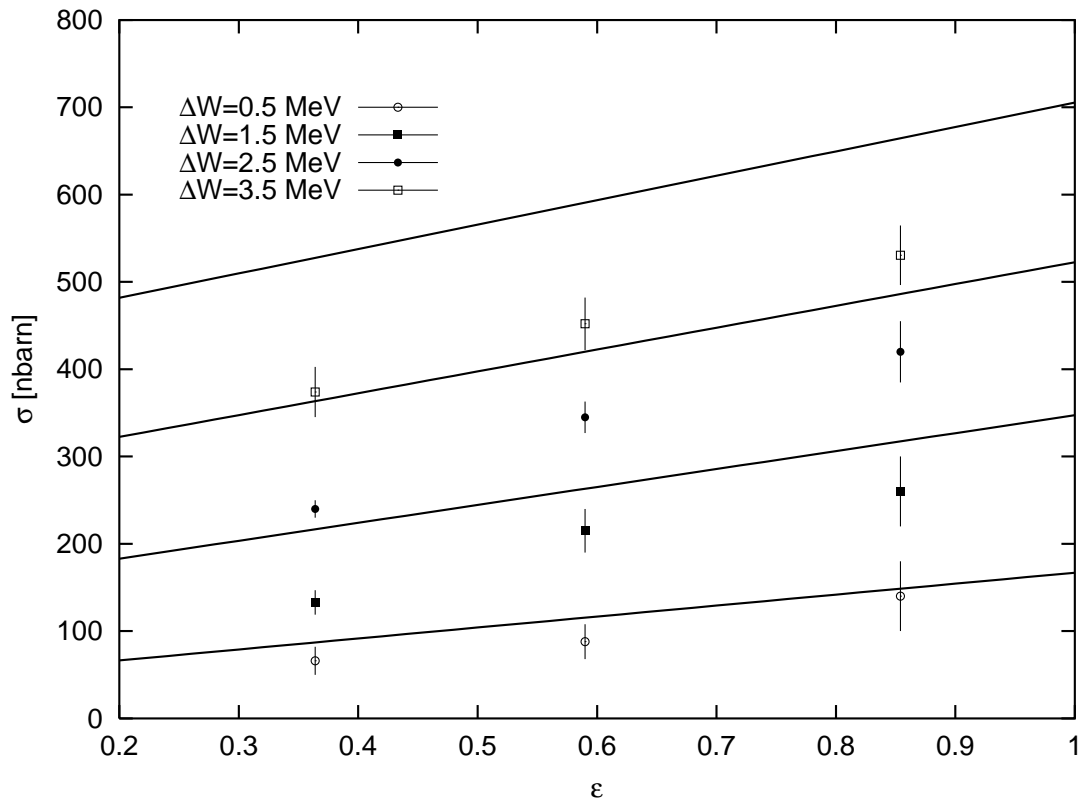

Figure 12: Total cross section as a function of the photon polarization $\varepsilon$ for four different values of the pion excess energy $\Delta W$ in comparison to the MAMI data [3] for fit 1 and the NNLO*-600 wave function. 


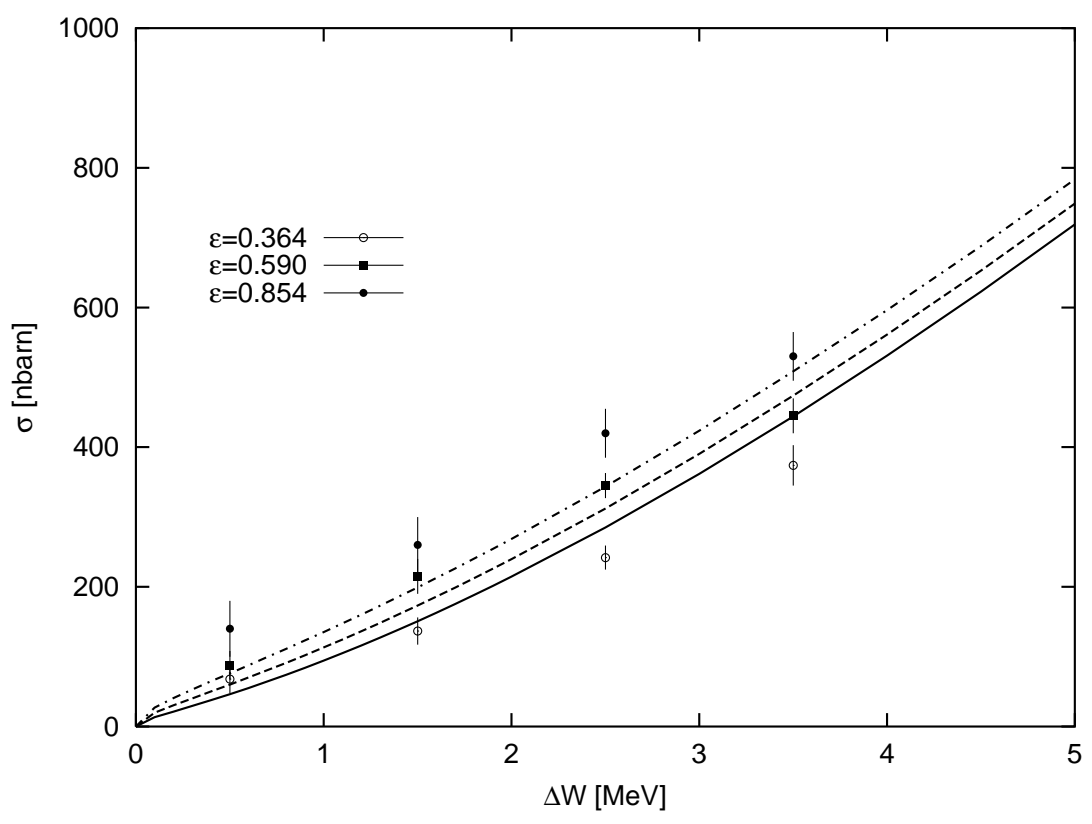

Figure 13: Total cross section as a function of $\Delta W$ for three different values of the photon polarization in comparison to the MAMI data [3] for fit 2 and the NNLO*-600 wave function.

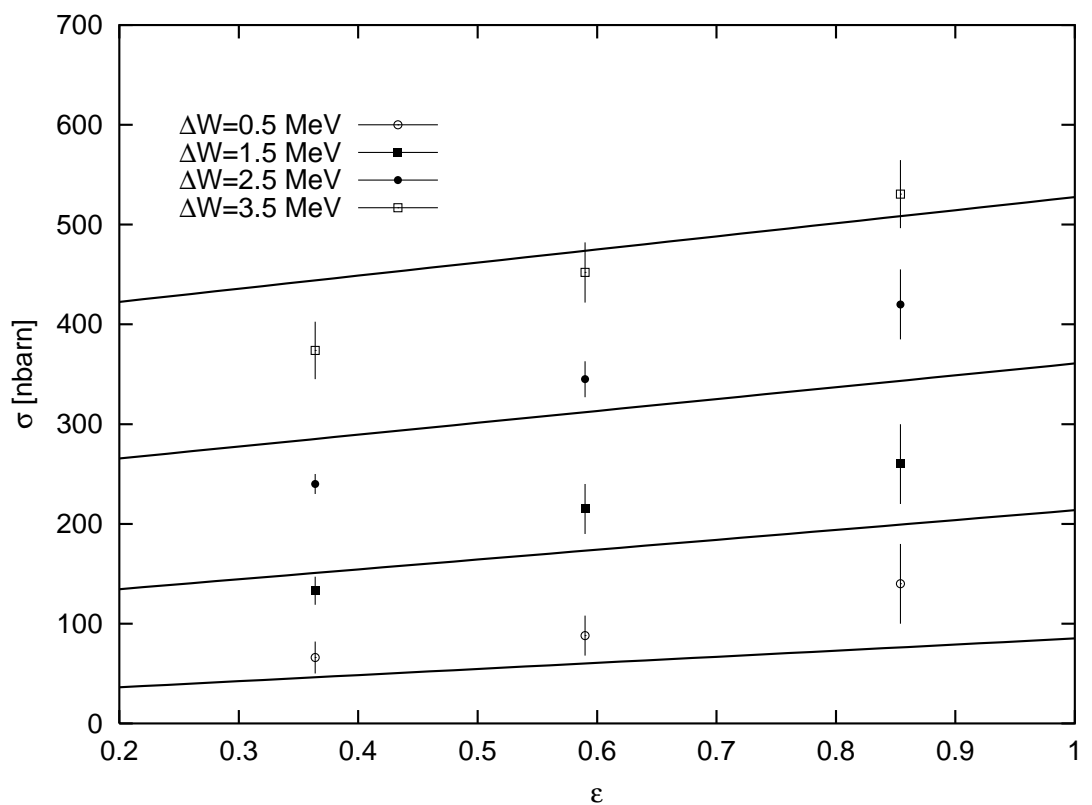

Figure 14: Total cross section as a function of the photon polarization $\varepsilon$ for four different values of the pion excess energy $\Delta W$ in comparison to the MAMI data [3] for fit 2 and the NNLO*-600 wave function. 
effects, our result for the transverse threshold multipole is consistent with the data. In Table 3 we collect the S-wave cross section $a_{0 d}$ for the various wave functions and fit procedures. We remark again that the scaled Swave cross section given in [2] was much too large because the dominant longitudinal multipole was not correctly represented. Thus, the dramatic difference between the CHPT prediction and the data has disappeared, and the overall description of the data is satisfactory but still needs to be improved. This will presumably be achieved when a complete fourth order calculation including the dominant isospin breaking effects has been performed.

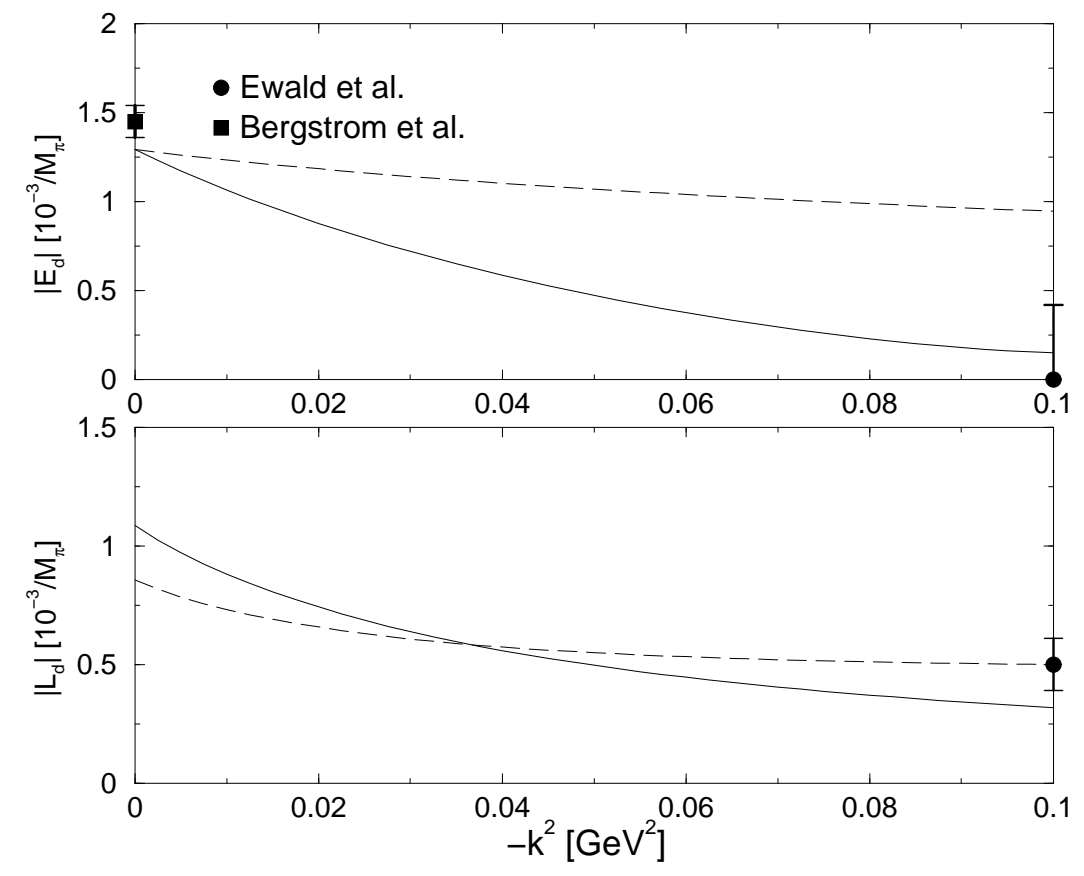

Figure 15: Threshold multipoles $\left|E_{d}\right|$ and $\left|L_{d}\right|$ as a function of the photon virtuality in comparison to the photon point data from SAL [26] and the electroproduction data from MAMI [3]. The sign of the experimental result for $L_{d}$ is taken to agree with the theoretical prediction. In our fits, the positive sign for $L_{d}$ is preferred. Solid (dashed) lines: Fit 2 (1). The NNLO*-600 wave function is used.

\section{Summary and conclusions}

In this paper, we have studied neutral pion electroproduction off deuterium in the framework of chiral perturbation theory at and above threshold. The salient ingredients and results of this work can be summarized as follows:

i) We have developed a general multipole decomposition for neutral pion production off spin-1 particles that is particularly suited for the threshold region and formulated in close analogy to the standard CGLN amplitudes for pion production off nucleons (spin-1/2 particles). Similar work was previously published in [8, 9].

ii) The interaction kernel and the wave functions are based consistently on chiral effective field theory. The kernel decomposes into a single scattering and a three-body contribution. We have chirally expanded the various contributions working to first non-trivial loop order $\mathcal{O}\left(q^{3}\right)$, with the exception of the S-waves for the single scattering contribution. These have to be included to fourth order with one additional fifth order term [10]. 


\begin{tabular}{|l||c|c|c|c|c|c|}
\hline$-k^{2}\left[\mathrm{GeV}^{2}\right]$ & 0.00 & 0.02 & 0.04 & 0.06 & 0.08 & 0.10 \\
\hline$a_{0 d}$ (NLO-500) [1] & 0.029 & 0.031 & 0.032 & 0.035 & 0.039 & 0.046 \\
$a_{0 d}$ (NLO-500) [2] & 0.029 & 0.022 & 0.016 & 0.014 & 0.013 & 0.013 \\
$a_{0 d}$ (NLO-600) [1] & 0.027 & 0.029 & 0.030 & 0.032 & 0.037 & 0.043 \\
$a_{0 d}$ (NLO-600) [2] & 0.027 & 0.020 & 0.016 & 0.013 & 0.013 & 0.014 \\
$a_{0 d}$ (NNLO*-500) [1] & 0.033 & 0.035 & 0.036 & 0.039 & 0.043 & 0.048 \\
$a_{0 d}$ (NNLO*-500) [2] & 0.033 & 0.024 & 0.018 & 0.014 & 0.013 & 0.012 \\
$a_{0 d}$ (NNLO*-600) [1] & 0.033 & 0.035 & 0.037 & 0.039 & 0.043 & 0.048 \\
$a_{0 d}$ (NNLO*-600) $[2]$ & 0.033 & 0.025 & 0.018 & 0.015 & 0.013 & 0.013 \\
\hline
\end{tabular}

Table 3: S-wave cross section $a_{0 d}$ in $\mu \mathrm{b}$ for the various wave functions (w.f.) and fit procedures $[n](n=1,2)$ employed.

iii) All parameters for pion production off the proton and the ones appearing in the three-body terms are fixed. The longitudinal neutron S-wave amplitude contains effectively two parameters, which we have determined by two different procedures. In the fits of type 1 we have fitted the fifth order parameter to the threshold multipole $L_{d}$ from Ref. [3] (and assuming resonance saturation to pin down the other LEC). The second procedure is based on a two parameter fit to the total cross section data from Ref.[3]. All results are completely insensitive to the wave functions used, showing that this reaction is sensitive to the long-range pion exchange firmly rooted in the chiral symmetry of QCD.

iv) The predicted differential cross sections are satisfactorily described for both fit procedures, although some systematic discrepancies for the higher values of the excess energy $\Delta W$ remain, see Figs. 9, 10. In particular, for fit 1 the total cross section rises too steeply with pion excess energy.

v) The calculated S- and $\mathrm{P}$-wave multipoles exhibit a more complex pion energy and photon virtuality dependence as assumed in the fits of Ref. 34. Within one standard deviation, the chiral predictions for the threshold multipoles $\left|E_{d}\right|$ and $\left|L_{d}\right|$ are consistent with the data at $k^{2}=0$ [26] and $k^{2}=-0.1 \mathrm{GeV}^{2}$ [3].

Clearly, the calculation presented here needs to be improved, in particular, the fourth order corrections to the $\mathrm{P}$-waves and the three-body terms have to be included (note that similar work for the $\mathrm{P}$-waves in neutral pion production off protons has only appeared recently [7]). However, we have demonstrated that chiral perturbation theory can be used successfully to analyze pion electroproduction data off the deuteron which gives access to the elementary neutron amplitude. It would be very interesting to also have data at lower photon virtuality, which might also help to resolve the mystery surrouding the proton data at $k^{2}=-0.05 \mathrm{GeV}^{2}$.

\section{Acknowledgements}

We are grateful to Christoph Hanhart for some useful comments.

\section{A Equivalence of multipole expansions}

In this appendix, we show the formal equivalence between the multipole expansion employed here and the earlier one developed in [9]. Our transition amplitude has the form (we suppress here the phase factor related to the $\varphi$ dependence)

$$
t_{m^{\prime}, \lambda, m}(\theta)=\sum_{L_{\pi}, L, J}\left\langle L_{\pi} \lambda+m-m^{\prime} 1 m^{\prime} \mid J \lambda+m\right\rangle\langle 1 m L \lambda \mid J \lambda+m\rangle \mathcal{O}_{L_{\pi}, J}^{L, \lambda} Y_{L_{\pi}, \lambda+m-m^{\prime}}(\theta, \varphi=0)
$$


in terms of the spherical harmonics. Arenhövel [9] works in the helicity basis and uses the rotation matrices $d_{M, M^{\prime}}^{J}(\theta)$,

$$
t_{m^{\prime}, \lambda, m}^{A}(\theta)=\sum_{L_{\pi}, L, J}\left\langle L_{\pi} 01-m^{\prime} \mid J-m^{\prime}\right\rangle\langle 1-m L \lambda \mid J \lambda-m\rangle \frac{\hat{L}_{\pi}}{\sqrt{4 \pi}} \mathcal{O}_{L_{\pi}, J}^{L, \lambda} d_{\lambda-m,-m^{\prime}}^{J}(\theta) .
$$

We now show that

$$
t_{m^{\prime}, \lambda, m}^{A}(\theta)=\sum_{\mu=-1}^{1} t_{-\mu, \lambda,-m}(\theta) d_{-\mu,-m^{\prime}}^{1}(\theta) .
$$

This can be proven simply by using the relation between the spherical harmonics and the d-functions,

$$
Y_{L_{\pi}, \lambda+m-m^{\prime}}(\theta, \varphi=0)=\frac{\hat{L}_{\pi}}{\sqrt{4 \pi}} d_{\lambda+m-m^{\prime}, 0}^{L_{\pi}}(\theta),
$$

and the following relation between rotation matrices

$$
\sum_{m_{1}, m_{2}}\left\langle j_{1} m_{1} j_{2} m_{2} \mid j m\right\rangle d_{m_{1}, m_{1}^{\prime}}^{j_{1}}(\theta) d_{m_{2}, m_{2}^{\prime}}^{j_{2}}(\theta)=\sum_{m^{\prime}}\left\langle j_{1} m_{1}^{\prime} j_{2} m_{2}^{\prime} \mid j m^{\prime}\right\rangle d_{m, m^{\prime}}^{j}(\theta) .
$$

With this, the equivalence between Eqs. (A.1) and (A.2) follows immediately.

\section{B Transformation matrices}

In this appendix, we collect the expressions of the various matrices appearing in Eqs. (2.13,2.14). For notational simplicity, we substitute the symbol for the pion angular momentum $L_{\pi}$ by $L$ in this appendix. Consider first $D_{L}$. The non-zero matrix elements are:

$$
\begin{aligned}
& D_{12}=\frac{\frac{i}{8}(-1+L)\left(P_{-2+L}-P_{L}\right)}{\sqrt{L}(-1+2 L)}, \quad D_{13}=\frac{\frac{-i}{8}(-1+L) P_{L}}{\sqrt{L}}, \quad D_{14}=\frac{\frac{-i}{8}(-1+L) P_{-1+L}}{\sqrt{L}}, \\
& D_{15}=\frac{-\left((1+L)\left(P_{-1+L}-P_{1+L}\right)\right)}{8 \sqrt{L}(1+2 L)}, \quad D_{16}=\frac{-\left((1+L)\left(P_{-2+L}-P_{L}\right)\right)}{16 \sqrt{L}(-1+2 L)}, \quad D_{18}=\frac{(1+L) P_{L}}{16 \sqrt{L}}, \\
& D_{19}=\frac{(1+L) P_{-1+L}}{16 \sqrt{L}}, \\
& D_{22}=\frac{-i \sqrt{\frac{-1+L}{L}} \sqrt{1+L}\left(P_{-2+L}-P_{L}\right)}{-8+16 L}, \quad D_{23}=\frac{i}{8} \sqrt{\frac{-1+L}{L}} \sqrt{1+L} P_{L}, \quad D_{24}=\frac{i}{8} \sqrt{\frac{-1+L}{L}} \sqrt{1+L} P_{-1+L}, \\
& D_{25}=\frac{\sqrt{\frac{-1+L}{L}} \sqrt{1+L}\left(-P_{-1+L}+P_{1+L}\right)}{8+16 L}, \quad D_{26}=\frac{\sqrt{\frac{-1+L}{L}} \sqrt{1+L}\left(-P_{-2+L}+P_{L}\right)}{-16+32 L}, \\
& D_{28}=\frac{\sqrt{\frac{-1+L}{L}} \sqrt{1+L} P_{L}}{16}, \quad D_{29}=\frac{\sqrt{\frac{-1+L}{L}} \sqrt{1+L} P_{-1+L}}{16}, \\
& D_{32}=\frac{i \sqrt{\frac{L}{1+L}} \sqrt{2+L}\left(P_{L}-P_{2+L}\right)}{24+16 L}, \quad D_{33}=\frac{-i}{8} \sqrt{\frac{L}{1+L}} \sqrt{2+L} P_{L}, \quad D_{34}=\frac{-i}{8} \sqrt{\frac{L}{1+L}} \sqrt{2+L} P_{1+L}, \\
& D_{35}=\frac{\sqrt{\frac{L}{1+L}} \sqrt{2+L}\left(P_{-1+L}-P_{1+L}\right)}{8+16 L}, \quad D_{36}=\frac{\sqrt{\frac{L}{1+L}} \sqrt{2+L}\left(P_{L}-P_{2+L}\right)}{48+32 L}, \\
& D_{38}=\frac{-\left(\sqrt{\frac{L}{1+L}} \sqrt{2+L} P_{L}\right)}{16}, \quad D_{39}=\frac{-\left(\sqrt{\frac{L}{1+L}} \sqrt{2+L} P_{1+L}\right)}{16}, \\
& D_{42}=\frac{\frac{-i}{8}(2+L)\left(P_{L}-P_{2+L}\right)}{\sqrt{1+L}(3+2 L)}, \quad D_{43}=\frac{\frac{i}{8}(2+L) P_{L}}{\sqrt{1+L}}, \quad D_{44}=\frac{\frac{i}{8}(2+L) P_{1+L}}{\sqrt{1+L}},
\end{aligned}
$$




$$
\begin{aligned}
& D_{45}=\frac{L\left(P_{-1+L}-P_{1+L}\right)}{8 \sqrt{1+L}(1+2 L)}, \quad D_{46}=\frac{L\left(P_{L}-P_{2+L}\right)}{16 \sqrt{1+L}(3+2 L)}, \quad D_{48}=\frac{-\left(L P_{L}\right)}{16 \sqrt{1+L}}, \quad D_{49}=\frac{-\left(L P_{1+L}\right)}{16 \sqrt{1+L}}, \\
& D_{55}=\frac{\sqrt{-2+L} \sqrt{L}(1+L)\left(P_{-1+L}-P_{1+L}\right)}{8 \sqrt{-1+2 L}(1+2 L)}, \quad D_{56}=\frac{\sqrt{-2+L} L^{\frac{3}{2}}\left(P_{-2+L}-P_{L}\right)}{8(-1+2 L)^{\frac{3}{2}}}, \\
& D_{57}=\frac{\sqrt{-2+L}(-1+L) \sqrt{L}\left(P_{-3+L}-P_{-1+L}\right)}{4(-3+2 L) \sqrt{-4+8 L}}, \quad D_{58}=\frac{-\left(\sqrt{-2+L} \sqrt{L} P_{L}\right)}{8 \sqrt{-1+2 L}}, \\
& D_{59}=\frac{-\left(\sqrt{-2+L} \sqrt{L} P_{-1+L}\right)}{8 \sqrt{-1+2 L}} \\
& D_{61}=\frac{\sqrt{L} \sqrt{1+L} \sqrt{-1+2 L}\left(P_{-1+L}-P_{1+L}\right)}{8+16 L}, \\
& D_{62}=\frac{\frac{i}{8}\left(\left(-3-2 L+3 L^{2}+2 L^{3}\right) P_{-2+L}+\left(3+4 L-6 L^{2}-4 L^{3}\right) P_{L}+L\left(-2+3 L+2 L^{2}\right) P_{2+L}\right)}{\sqrt{\frac{L}{1+L}} \sqrt{-1+2 L}(1+2 L)(3+2 L)}, \\
& D_{63}=\frac{\frac{i}{8} \sqrt{-1+2 L} P_{L}}{\sqrt{\frac{L}{1+L}}}, \quad D_{64}=\frac{\frac{i}{8} \sqrt{-1+2 L}\left((1+L) P_{-1+L}+L P_{1+L}\right)}{\sqrt{\frac{L}{1+L}}(1+2 L)}, \\
& D_{65}=\frac{-\left(\left(-3+L+L^{2}\right)\left(P_{-1+L}-P_{1+L}\right)\right)}{24 \sqrt{\frac{L}{1+L}} \sqrt{-1+2 L}(1+2 L)} \text {, } \\
& D_{66}=\frac{-\left((3+2 L)^{2}\left(-1+L^{2}\right) P_{-2+L}\right)+\left(-9-14 L+12 L^{2}+8 L^{3}\right) P_{L}+(1-2 L)^{2} L(2+L) P_{2+L}}{48 \sqrt{\frac{L}{1+L}}(-1+2 L)^{\frac{3}{2}}(1+2 L)(3+2 L)}, \\
& D_{67}=\frac{\sqrt{L}(1+L)^{\frac{3}{2}}\left(-P_{-1+L}+P_{1+L}\right)}{24 \sqrt{-1+2 L}(1+2 L)}, \quad D_{68}=\frac{-P_{L}}{16 \sqrt{\frac{L}{1+L}} \sqrt{-1+2 L}} \\
& D_{69}=\frac{-\left(\left(3+5 L+2 L^{2}\right) P_{-1+L}+(1-2 L) L P_{1+L}\right)}{48 \sqrt{\frac{L}{1+L}} \sqrt{-1+2 L}(1+2 L)}, \\
& D_{71}=\frac{\sqrt{L} \sqrt{1+L}\left(-P_{-1+L}+P_{1+L}\right)}{8 \sqrt{1+2 L}} \\
& D_{72}=\frac{\frac{-i}{8}\left(\left(-3-2 L+3 L^{2}+2 L^{3}\right) P_{-2+L}+\left(3+4 L-6 L^{2}-4 L^{3}\right) P_{L}+L\left(-2+3 L+2 L^{2}\right) P_{2+L}\right)}{\sqrt{L} \sqrt{1+L} \sqrt{1+2 L}\left(-3+4 L+4 L^{2}\right)}, \\
& D_{73}=\frac{\frac{-i}{8} \sqrt{1+2 L} P_{L}}{\sqrt{L} \sqrt{1+L}}, \quad D_{74}=\frac{\frac{-i}{8}\left((1+L) P_{-1+L}+L P_{1+L}\right)}{\sqrt{L} \sqrt{1+L} \sqrt{1+2 L}}, \\
& D_{75}=\frac{-\left(\left(-3+L+L^{2}\right)\left(P_{-1+L}-P_{1+L}\right)\right)}{24 \sqrt{L} \sqrt{1+L} \sqrt{1+2 L}}, \\
& D_{76}=\frac{-\left((3+2 L)^{2}\left(-1+L^{2}\right) P_{-2+L}\right)+\left(-9-14 L+12 L^{2}+8 L^{3}\right) P_{L}+(1-2 L)^{2} L(2+L) P_{2+L}}{48 \sqrt{L} \sqrt{1+L} \sqrt{1+2 L}\left(-3+4 L+4 L^{2}\right)}, \\
& D_{77}=\frac{\sqrt{L} \sqrt{1+L}\left(-P_{-1+L}+P_{1+L}\right)}{24 \sqrt{1+2 L}}, \quad D_{78}=\frac{-\left(\sqrt{1+2 L} P_{L}\right)}{16 \sqrt{L} \sqrt{1+L}}, \\
& D_{79}=\frac{-\left(\left(3+5 L+2 L^{2}\right) P_{-1+L}+(1-2 L) L P_{1+L}\right)}{48 \sqrt{L} \sqrt{1+L} \sqrt{1+2 L}}, \\
& D_{81}=\frac{\sqrt{L} \sqrt{1+L} \sqrt{3+2 L}\left(P_{-1+L}-P_{1+L}\right)}{8+16 L},
\end{aligned}
$$




$$
\begin{aligned}
& D_{82}=\frac{\frac{-i}{8} \sqrt{\frac{L}{1+L}}\left(\left(-3-2 L+3 L^{2}+2 L^{3}\right) P_{-2+L}+\left(3+4 L-6 L^{2}-4 L^{3}\right) P_{L}+L\left(-2+3 L+2 L^{2}\right) P_{2+L}\right)}{\sqrt{3+2 L}\left(-1+4 L^{2}\right)}, \\
& D_{83}=\frac{-i}{8} \sqrt{\frac{L}{1+L}} \sqrt{3+2 L} P_{L}, \quad D_{84}=\frac{-i \sqrt{\frac{L}{1+L}} \sqrt{3+2 L}\left((1+L) P_{-1+L}+L P_{1+L}\right)}{8+16 L}, \\
& D_{85}=\frac{-\left(\sqrt{\frac{L}{1+L}}\left(-3+L+L^{2}\right)\left(P_{-1+L}-P_{1+L}\right)\right)}{24(1+2 L) \sqrt{3+2 L}}, \\
& D_{86}=\frac{-\left(\sqrt{\frac{L}{1+L}}\left((3+2 L)^{2}\left(-1+L^{2}\right) P_{-2+L}+\left(9+14 L-12 L^{2}-8 L^{3}\right) P_{L}-(1-2 L)^{2} L(2+L) P_{2+L}\right)\right)}{48(3+2 L)^{\frac{3}{2}}\left(-1+4 L^{2}\right)}, \\
& D_{87}=\frac{L^{\frac{3}{2}} \sqrt{1+L}\left(-P_{-1+L}+P_{1+L}\right)}{24(1+2 L) \sqrt{3+2 L}}, \quad D_{88}=\frac{-\left(\sqrt{\frac{L}{1+L}} P_{L}\right)}{16 \sqrt{3+2 L}}, \\
& D_{89}=\frac{-\left(\sqrt{\frac{L}{1+L}}\left(\left(3+5 L+2 L^{2}\right) P_{-1+L}+(1-2 L) L P_{1+L}\right)\right)}{48(1+2 L) \sqrt{3+2 L}}, \\
& D_{95}=\frac{L \sqrt{1+L} \sqrt{3+L}\left(P_{-1+L}-P_{1+L}\right)}{8(1+2 L) \sqrt{3+2 L}}, \quad D_{96}=\frac{(1+L)^{\frac{3}{2}} \sqrt{3+L}\left(P_{L}-P_{2+L}\right)}{8(3+2 L)^{\frac{3}{2}}}, \\
& D_{97}=\frac{\sqrt{1+L}(2+L) \sqrt{3+L}\left(P_{1+L}-P_{3+L}\right)}{8 \sqrt{3+2 L}(5+2 L)}, \quad D_{98}=\frac{\sqrt{1+L} \sqrt{3+L} P_{L}}{8 \sqrt{3+2 L}}, \\
& D_{99}=\frac{\sqrt{1+L} \sqrt{3+L} P_{1+L}}{8 \sqrt{3+2 L}} .
\end{aligned}
$$

Here, the $P_{L}$ are the conventional Legendre polynomials that depend on $z=\cos \theta$. Note that $L$ is positive definite so that Legendre polynomials with negative index have to be understood as zero. The matrix $E_{L}$ has no zero elements and takes the form

$$
E_{L}=\left(\begin{array}{cccc}
\frac{\frac{-i}{4} \sqrt{-1+L} P_{L}}{\sqrt{2}} & \frac{\frac{-i}{4} \sqrt{-1+L} P_{-1+L}}{\sqrt{2}} & \frac{\sqrt{-1+L}(1+L)\left(P_{-1+L}-P_{1+L}\right)}{8 \sqrt{2}(1+2 L)} & \frac{\sqrt{-1+L}(1+L)\left(P_{-2+L}-P_{L}\right)}{8 \sqrt{2}(-1+2 L)} \\
\frac{\frac{i}{4} \sqrt{1+L} P_{L}}{\sqrt{2}} & \frac{\frac{i}{4} \sqrt{1+L} P_{-1+L}}{\sqrt{2}} & \frac{(-1+L) \sqrt{1+L}\left(P_{-1+L}-P_{1+L}\right)}{8 \sqrt{2}(1+2 L)} & \frac{(-1+L) \sqrt{1+L}\left(P_{-2+L}-P_{L}\right)}{8 \sqrt{2}(-1+2 L)} \\
\frac{\frac{i}{4} \sqrt{L} P_{L}}{\sqrt{2}} & \frac{\frac{i}{4} \sqrt{L} P_{1+L}}{\sqrt{2}} & \frac{-\left(\sqrt{L}(2+L)\left(P_{-1+L}-P_{1+L}\right)\right)}{8 \sqrt{2}(1+2 L)} & \frac{-\left(\sqrt{L}(2+L)\left(P_{L}-P_{2+L}\right)\right)}{8 \sqrt{2}(3+2 L)} \\
\frac{\frac{-i}{4} \sqrt{2+L} P_{L}}{\sqrt{2}} & \frac{\frac{-i}{4} \sqrt{2+L} P_{1+L}}{\sqrt{2}} & \frac{-\left(L \sqrt{2+L}\left(P_{-1+L}-P_{1+L}\right)\right)}{8 \sqrt{2}(1+2 L)} & \frac{-\left(L \sqrt{2+L}\left(P_{L}-P_{2+L}\right)\right)}{8 \sqrt{2}(3+2 L)}
\end{array}\right) .
$$

We now turn to the matrices $G_{L_{\pi}}$ and $H_{L_{\pi}}$ appearing in the inverse transformation, Eq. (2.14). It is most convenient to express these with the help of Clebsch-Gordan coefficients. For that, we employ the $D$-symbols

$$
D_{\lambda, m^{\prime}, m}^{L, L_{\pi}, J}=\left\langle L_{\pi} \lambda+m-m^{\prime} 1 m^{\prime} \mid J \lambda+m\right\rangle\langle 1 m L \lambda \mid J \lambda+m\rangle,
$$

as they appear also in the multipole expansion of the $T$-matrix. We define the transverse and the longitudinal vectors $T\left(L, L_{\pi}, J\right)$ and $L\left(L, L_{\pi}, J\right)$, respectively, in terms of their components:

$$
\begin{aligned}
& T\left(L, L_{\pi}, J\right)_{1}=\frac{4 \sqrt{1+2 L} \sqrt{1+2 L_{\pi}}\left(D_{1,-1,-1}^{L, L_{\pi}, J}+D_{1,0,0}^{L, L_{\pi}, J}+D_{1,1,1}^{L, L_{\pi}, J}\right) P_{L_{\pi}}^{(1)}}{3 \sqrt{1+2 J} \sqrt{L_{\pi}\left(1+L_{\pi}\right)}}, \\
& T\left(L, L_{\pi}, J\right)_{2}=\frac{2 i \sqrt{2+4 L} \sqrt{1+2 L_{\pi}}\left(D_{1,-1,0}^{L, L_{\pi}, J}+D_{1,0,1}^{L, L_{\pi}, J}\right) P_{L_{\pi}}^{(2)}}{\sqrt{1+2 J} \sqrt{L_{\pi}\left(1+L_{\pi}\right)} \sqrt{-2+L_{\pi}+L_{\pi}^{2}}}, \\
& T\left(L, L_{\pi}, J\right)_{3}=\frac{i \sqrt{2+4 L} \sqrt{1+2 L_{\pi}}\left(D_{1,0,-1}^{L, L_{\pi}, J}+D_{1,1,0}^{L, L_{\pi}, J}\right) P_{L_{\pi}}}{\sqrt{1+2 J}}
\end{aligned}
$$




$$
\begin{aligned}
& +\frac{2 i \sqrt{1+2 L} \sqrt{1+2 L_{\pi}} z\left(D_{1,-1,-1}^{L, L_{\pi}, J}-D_{1,1,1}^{L, L_{\pi}, J}\right) P_{L_{\pi}}^{(1)}}{\sqrt{1+2 J} \sqrt{L_{\pi}\left(1+L_{\pi}\right)}} \\
& -\frac{i \sqrt{2+4 L} \sqrt{1+2 L_{\pi}}\left(-1+z^{2}\right)\left(D_{1,-1,0}^{L, L_{\pi}, J}+D_{1,0,1}^{L, L_{\pi}, J}\right) P_{L_{\pi}}^{(2)}}{\sqrt{1+2 J} \sqrt{L_{\pi}\left(1+L_{\pi}\right)} \sqrt{-2+L_{\pi}+L_{\pi}^{2}}}, \\
& T\left(L, L_{\pi}, J\right)_{4}=-\frac{2 i \sqrt{1+2 L} \sqrt{1+2 L_{\pi}}\left(D_{1,-1,-1}^{L, L_{\pi}, J}-D_{1,1,1}^{L, L_{\pi}, J}\right) P_{L_{\pi}}^{(1)}}{\sqrt{1+2 J} \sqrt{L_{\pi}\left(1+L_{\pi}\right)}}, \\
& T\left(L, L_{\pi}, J\right)_{5}=\frac{2 \sqrt{1+2 L} \sqrt{1+2 L_{\pi}}}{\sqrt{1+2 J} \sqrt{L_{\pi}\left(1+L_{\pi}\right)}}\left(D_{1,-1,-1}^{L, L_{\pi}, J}-2 D_{1,0,0}^{L, L_{\pi}, J}-D_{1,1,-1}^{L, L_{\pi}, J}+D_{1,1,1}^{L, L_{\pi}, J}\right) P_{L_{\pi}}^{(1)} \\
& +\frac{4 \sqrt{2+4 L} \sqrt{1+2 L_{\pi}} z\left(-D_{1,-1,0}^{L, L_{\pi}, J}+D_{1,0,1}^{L, L_{\pi}, J}\right) P_{L_{\pi}}^{(2)}}{\sqrt{1+2 J} \sqrt{L_{\pi}\left(1+L_{\pi}\right)} \sqrt{-2+L_{\pi}+L_{\pi}^{2}}} \\
& +\frac{2 \sqrt{1+2 L} \sqrt{1+2 L_{\pi}}\left(1+3 z^{2}\right) D_{1,-1,1}^{L, L_{\pi}, J} P_{L_{\pi}}^{(3)}}{\sqrt{1+2 J} \sqrt{L_{\pi}\left(1+L_{\pi}\right)} \sqrt{-6+L_{\pi}+L_{\pi}^{2}} \sqrt{-2+L_{\pi}+L_{\pi}^{2}}}, \\
& T\left(L, L_{\pi}, J\right)_{6}=\frac{4 \sqrt{2+4 L} \sqrt{1+2 L_{\pi}}\left(D_{1,-1,0}^{L, L_{\pi}, J}-D_{1, L_{\pi}, 1}^{L, L_{\pi}}\right) P_{L_{\pi}}^{(2)}}{\sqrt{1+2 J} \sqrt{L_{\pi}\left(1+L_{\pi}\right)} \sqrt{-2+L_{\pi}+L_{\pi}^{2}}} \\
& -\frac{16 \sqrt{1+2 L} \sqrt{1+2 L_{\pi}} z D_{1,-1,1}^{L, L_{\pi}, J} P_{L_{\pi}}^{(3)}}{\sqrt{1+2 J} \sqrt{L_{\pi}\left(1+L_{\pi}\right)} \sqrt{-6+L_{\pi}+L_{\pi}^{2}} \sqrt{-2+L_{\pi}+L_{\pi}^{2}}}, \\
& T\left(L, L_{\pi}, J\right)_{7}=\frac{8 \sqrt{1+2 L} \sqrt{1+2 L_{\pi}} D_{1,-1,1}^{L, L_{\pi}, J} P_{L_{\pi}}^{(3)}}{\sqrt{1+2 J} \sqrt{L_{\pi}\left(1+L_{\pi}\right)} \sqrt{-6+L_{\pi}+L_{\pi}^{2}} \sqrt{-2+L_{\pi}+L_{\pi}^{2}}}, \\
& T\left(L, L_{\pi}, J\right)_{8}=\frac{2 \sqrt{2+4 L} \sqrt{1+2 L_{\pi}}\left(D_{1,0,-1}^{L, L_{\pi}, J}-D_{1,1,0}^{L, L_{\pi}, J}\right) P_{L_{\pi}}}{\sqrt{1+2 J}} \\
& +\frac{4 \sqrt{1+2 L} \sqrt{1+2 L_{\pi}} z D_{1,1,-1}^{L, L_{\pi}, J} P_{L_{\pi}}^{(1)}}{\sqrt{1+2 J} \sqrt{L_{\pi}\left(1+L_{\pi}\right)}} \\
& +\frac{2 \sqrt{2+4 L} \sqrt{1+2 L_{\pi}}\left(-1+z^{2}\right)\left(D_{1,-1,0}^{L, L_{\pi}, J}-D_{1,0,1}^{L, L_{\pi}, J}\right) P_{L_{\pi}}^{(2)}}{\sqrt{1+2 J} \sqrt{L_{\pi}\left(1+L_{\pi}\right)} \sqrt{-2+L_{\pi}+L_{\pi}^{2}}} \\
& -\frac{4 \sqrt{1+2 L} \sqrt{1+2 L_{\pi}} z\left(-1+z^{2}\right) D_{1,-1,1}^{L, L_{\pi},} P_{L_{\pi}}^{(3)}}{\sqrt{1+2 J} \sqrt{L_{\pi}\left(1+L_{\pi}\right)} \sqrt{-6+L_{\pi}+L_{\pi}^{2}} \sqrt{-2+L_{\pi}+L_{\pi}^{2}}}, \\
& T\left(L, L_{\pi}, J\right)_{9}=\frac{-4 \sqrt{1+2 L} \sqrt{1+2 L_{\pi}} D_{1,1,-1}^{L, L_{\pi}, J} P_{L_{\pi}}^{(1)}}{\sqrt{1+2 J} \sqrt{L_{\pi}\left(1+L_{\pi}\right)}} \\
& +\frac{4 \sqrt{1+2 L} \sqrt{1+2 L_{\pi}}\left(-1+z^{2}\right) D_{1,-1,1}^{L, L_{\pi}, J} P_{L_{\pi}}^{(3)}}{\sqrt{1+2 J} \sqrt{L_{\pi}\left(1+L_{\pi}\right)} \sqrt{-6+L_{\pi}+L_{\pi}^{2}} \sqrt{-2+L_{\pi}+L_{\pi}^{2}}} ; \\
& L\left(L, L_{\pi}, J\right)_{1}=\frac{2 i \sqrt{2+4 L} \sqrt{1+2 L_{\pi}} D_{0,1,1}^{L, L_{\pi}, J} P_{L_{\pi}}}{\sqrt{1+2 J}} \\
& +\frac{2 i \sqrt{1+2 L} \sqrt{1+2 L_{\pi}} z\left(D_{0,0,1}^{L, L_{\pi}, J}-D_{0,1,0}^{L, L_{\pi}, J}\right) P_{L_{\pi}}^{(1)}}{\sqrt{1+2 J} \sqrt{L_{\pi}\left(1+L_{\pi}\right)}}, \\
& L\left(L, L_{\pi}, J\right)_{2}=\frac{-2 i \sqrt{1+2 L} \sqrt{1+2 L_{\pi}}\left(D_{0,0,1}^{L, L_{\pi}, J}-D_{0,1,0}^{L, L_{\pi}, J}\right) P_{L_{\pi}}^{(1)}}{\sqrt{1+2 J} \sqrt{L_{\pi}\left(1+L_{\pi}\right)}}, \\
& L\left(L, L_{\pi}, J\right)_{3}=\frac{-4 \sqrt{1+2 L} \sqrt{1+2 L_{\pi}}\left(D_{0,0,1}^{L, L_{\pi}, J}+D_{0,1,0}^{L, L_{\pi}, J}\right) P_{L_{\pi}}^{(1)}}{\sqrt{1+2 J} \sqrt{L_{\pi}\left(1+L_{\pi}\right)}}
\end{aligned}
$$




$$
\begin{aligned}
&+ \frac{4 \sqrt{2+4 L} \sqrt{1+2 L_{\pi}} z D_{0,1,-1}^{L, L_{\pi}, J} P_{L_{\pi}}^{(2)}}{\sqrt{1+2 J} \sqrt{L_{\pi}\left(1+L_{\pi}\right)} \sqrt{-2+L_{\pi}+L_{\pi}^{2}}}, \\
& L\left(L, L_{\pi}, J\right)_{4}=\frac{-4 \sqrt{2+4 L} \sqrt{1+2 L_{\pi}} D_{0,1,-1}^{L, L_{\pi}, J} P_{L_{\pi}}^{(2)}}{\sqrt{1+2 J} \sqrt{L_{\pi}\left(1+L_{\pi}\right)} \sqrt{-2+L_{\pi}+L_{\pi}^{2}}}
\end{aligned}
$$

where $P_{L}^{(n)}(z)$ is the $n^{\text {th }}$ derivative of the Legendre polynom $P_{L}(z)$. The matrices $G_{L_{\pi}}$ and $H_{L_{\pi}}$ can then be expressed in terms of the following vectors:

$$
\begin{aligned}
G_{L_{\pi}} & =\left(G_{1}, \ldots, G_{9}\right), \\
H_{L_{\pi}} & =\left(H_{1}, \ldots, H_{4}\right),
\end{aligned}
$$

with

$$
\begin{aligned}
& G_{1}=T\left(L_{\pi}-1, L_{\pi}, L_{\pi}-1\right), \quad G_{2}=T\left(L_{\pi}-1, L_{\pi}, L_{\pi}\right), \quad G_{3}=T\left(L_{\pi}+1, L_{\pi}, L_{\pi}\right), \\
& G_{4}=T\left(L_{\pi}+1, L_{\pi}, L_{\pi}+1\right), \quad G_{5}=T\left(L_{\pi}-2, L_{\pi}, L_{\pi}-1\right), \quad G_{6}=T\left(L_{\pi}, L_{\pi}, L_{\pi}-1\right), \\
& G_{7}=T\left(L_{\pi}, L_{\pi}, L_{\pi}\right), \quad G_{8}=T\left(L_{\pi}, L_{\pi}, L_{\pi}+1\right), \quad G_{9}=T\left(L_{\pi}+2, L_{\pi}, L_{\pi}+1\right),
\end{aligned}
$$

and

$$
\begin{aligned}
& H_{1}=L\left(L_{\pi}-1, L_{\pi}, L_{\pi}-1\right), \quad H_{2}=L\left(L_{\pi}-1, L_{\pi}, L_{\pi}\right), \\
& H_{3}=L\left(L_{\pi}+1, L_{\pi}, L_{\pi}\right), \quad H_{4}=L\left(L_{\pi}+1, L_{\pi}, L_{\pi}+1\right) .
\end{aligned}
$$

\section{Two-body to three-body center-of-mass}

In this appendix we sketch the derivation of the transformation from the $\gamma$ - $d$ center-of-mass (COM) system to the $\gamma-N$ COM, extending the considerations given in [16]. We are interested in the kinematics of the process $\gamma^{*} N_{1} N_{2} \rightarrow \pi N_{1} N_{2}$, where the nucleons, $N_{1}$ and $N_{2}$, are sewn to the deuteron wavefunctions. Our 3-body corrections are evaluated in the $\gamma-d$ COM whereas the single scattering corrections which take into account the scattering of the photon on the individual nucleons have been calculated in the $\gamma-N$ COM. It is therefore necessary to construct the Lorentz transformation which boosts the single-scattering corrections to the $\gamma-d$ COM.

Let $p$ be some four-vector in the $\gamma-d \mathrm{COM}$ and $p^{*}$ the corresponding four-vector in the COM of the (second) single nucleon. These are related by the Lorentz transformation $p^{*}=\Lambda(\vec{u}) p$ with $\vec{u}=u \vec{e}_{x}$ the velocity. The vector $p$ transforms as

$$
\left(\begin{array}{c}
p_{0}^{*} \\
p_{\|}^{*}
\end{array}\right)=\left(\begin{array}{cc}
\gamma & -\beta \gamma \\
-\beta \gamma & \gamma
\end{array}\right)\left(\begin{array}{l}
p_{0} \\
p_{\|}
\end{array}\right),
$$

and the transverse directions are of course unaffected. This gives

$$
\vec{\beta}=\frac{\vec{p}_{2}+\vec{k}}{k_{0}+p_{20}}=-\frac{\vec{p}_{1}}{k_{0}+p_{20}},
$$

so that $\vec{e}_{x}=-\hat{p}_{1}$. The photon energy-momentum four-vector thus transforms as

$$
\begin{aligned}
k_{0}^{*} & =\gamma\left(k_{0}+\beta \vec{k} \cdot \hat{p}_{1}\right), \\
\vec{k}^{*} & =\vec{k}-\left(\vec{k} \cdot \hat{p}_{1}(1-\gamma)-\gamma \beta k_{0}\right) \hat{p}_{1} .
\end{aligned}
$$

Expanded in powers of $1 / m$, this reads

$$
\begin{aligned}
k_{0}^{*} & =k_{0}+\frac{1}{m} \vec{k} \cdot \vec{p}_{1}+\mathcal{O}\left(1 / m^{2}\right), \\
\vec{k}^{*} & =\vec{k}+\frac{1}{m} k_{0} \vec{p}_{1}+\mathcal{O}\left(1 / m^{2}\right),
\end{aligned}
$$


and similarly for the pion energy and three-momentum $\left(q_{0}=\omega, \vec{q}\right)$. One also has to transform the photon polarization vector. This is most easily done if one uses the following gauge-invariant form of the $\gamma^{*} N \rightarrow \pi^{0} N$ transition amplitude,

$$
\mathcal{M}_{\mathrm{ss}}=\sum_{i=1}^{6} \mathcal{O}_{\mathrm{ss}, i} F_{\mathrm{ss}, i}^{*},
$$

with

$$
\begin{aligned}
& \mathcal{O}_{\mathrm{ss}, 1}=\vec{\varepsilon}^{*} \cdot\left(\hat{k}^{*} \times \hat{q}^{*}\right), \quad \mathcal{O}_{\mathrm{ss}, 2}=\vec{\varepsilon}^{*} \cdot\left(\hat{k}^{*} \times \hat{q}^{*}\right) \vec{S} \cdot\left(\hat{k}^{*} \times \hat{q}^{*}\right), \quad \mathcal{O}_{\mathrm{ss}, 3}=\vec{\varepsilon}^{*} \cdot\left(\hat{k}^{*} \times\left(\hat{k}^{*} \times \vec{S}\right)\right), \\
& \mathcal{O}_{\mathrm{ss}, 4}=\vec{\varepsilon}^{*} \cdot\left(\hat{k}^{*} \times \hat{q}^{*}\right) \vec{S} \cdot\left(\hat{k}^{*} \times \hat{q}^{*}\right), \mathcal{O}_{\mathrm{ss}, 5}=\left(\vec{\varepsilon}^{*} \cdot \hat{k}^{*}-\frac{k^{*}}{k_{0}^{*}} \varepsilon_{0}^{*}\right) \vec{S} \cdot \hat{k}^{*}, \mathcal{O}_{\mathrm{ss}, 6}=\left(\vec{\varepsilon}^{*} \cdot \hat{k}^{*}-\frac{k^{*}}{k_{0}^{*}} \varepsilon_{0}^{*}\right) \vec{S} \cdot \hat{q}^{*},
\end{aligned}
$$

Since we work in the Coulomb gauge in the $\gamma-d$ COM, the full Lorentz transformation for the polarization vector is given by

$$
\varepsilon_{0}^{*}=\gamma \beta \vec{\varepsilon} \cdot \hat{p}_{1}, \quad \vec{\varepsilon}^{*}=\vec{\varepsilon}-\vec{\varepsilon} \cdot \hat{p}_{1}(1-\gamma) \hat{p}_{1} .
$$

Expanded in powers of $1 / m$, one observes that only the time-component is modified to leading order,

$$
\varepsilon_{0}^{*}=\frac{1}{m} \vec{\varepsilon} \cdot \vec{p}_{1}+\mathcal{O}\left(1 / m^{2}\right), \quad \vec{\varepsilon}^{*}=\vec{\varepsilon}+\mathcal{O}\left(1 / m^{2}\right) .
$$

\section{References}

[1] Ulf-G. Meißner, "Pion production in chiral perturbation theory," in proceedings of the workshop on "Chiral fluctuations in hadronic matter," IPN Orsay, France, September 26-28, 2001.

[2] V. Bernard, H. Krebs and Ulf-G. Meißner, Phys. Rev. C61 (2000) 058201 arXiv:nucl-th/9912033.

[3] I. Ewald et al., Phys. Lett. B499 (2001) 238 arXiv:nucl-ex/0010008].

[4] H. Merkel et al., Phys. Rev. Lett. 88 (2002) 012301 arXiv:nucl-ex/0108020].

[5] H.B. van den Brink et al., Phys. Rev. Lett. 74 (1995) 3561; Nucl. Phys. A612 (1997) 391.

[6] M.O. Distler et al., Phys. Rev. Lett. 80 (1998) 2294.

[7] V. Bernard, N. Kaiser and Ulf-G. Meißner, Eur. Phys. J. A11 (2001) 209 arXiv:hep-ph/0102066.

[8] H. Arenhövel, Few Body Syst. 25 (1998) 127 [arXiv:nucl-th/9809067.

[9] H. Arenhövel, Few Body Syst. 27 (1999) 141 arXiv:nucl-th/9907005.

[10] V. Bernard, N. Kaiser and Ulf-G. Meißner, Nucl. Phys. A607 (1996) 379 [Erratum-ibid. A633 (1996) 695] arXiv:hep-ph/9601267.

[11] E. Epelbaum, Ulf-G. Meißner and W. Glöckle, Nucl. Phys. A671 (2000) 295 arXiv:nucl-th/9910064.

[12] E. Epelbaum, Ulf-G. Meißner, W. Glöckle and C. Elster, Phys. Rev. C65 (2001), 044001 arXiv:nucl th/0106007.

[13] E. Epelbaum, A. Nogga, W. Glöckle, H. Kamada, Ulf-G. Meißner and H. Witala, arXiv:nucl-th/0201064, accepted for publication in Eur. Phys. J. A.

[14] L. D. Pearlstein and A. Klein, Phys. Rev. 107 (1957) 836.

[15] S. R. Beane, C. Y. Lee and U. van Kolck, Phys. Rev. C52 (1995) 2914 arXiv:nucl-th/9506017.

[16] S. R. Beane, V. Bernard, T.-S.H. Lee, Ulf-G. Meißner and U. van Kolck, Nucl. Phys. A618 (1997) 381 arXiv:hep-ph/9702226.

[17] V. Bernard, N. Kaiser and Ulf-G. Meißner, Int. J. Mod. Phys. E4 (1995) 193 arXiv:hep-ph/9501384.

[18] S. Weinberg, Nucl. Phys. B363 (1991) 3. 
[19] S. Weinberg, Phys. Lett. B295 (1992) 114.

[20] G. F. Chew, M. L. Goldberger, F. E. Low and Y. Nambu, Phys. Rev. 106 (1957) 1345.

[21] F. A. Berends, A. Donnachie and D. L. Weaver, Nucl. Phys. B4 (1967) 1.

[22] J.H. Koch and R.M. Woloshyn, Phys. Rev. C16 (1977) 1968.

[23] V. Bernard, N. Kaiser and Ulf-G. Meißner, Z. Phys. C70 (1996) 483 [arXiv:hep-ph/9411287.

[24] V. Bernard, N. Kaiser and Ulf-G. Meißner, $\pi$ N Newsletter 7 (1992) 62.

[25] M. Rekalo and E. Tomasi-Gustafsson, Phys. Rev. C66 (2002) 015203 arXiv:nucl-th/0112063.

[26] J.C. Bergstrom et al., Phys. Rev. C57 (1998) 3203. 